\title{
Parallel algorithm for convection- diffusion system based on least-squares procedure
}

\author{
Jiansong Zhang ${ }^{1 *}$, Hui Guo ${ }^{2}$, Hongfei Fu ${ }^{3}$ and Yanzhen Chang ${ }^{4}$
}

${ }^{*}$ Correspondence:
jszhang@upc.edu.cn
${ }^{1}$ Department of Applied
Mathematics, China
University of Petroleum,
Qingdao 266580, China
Full list of author information
is available at the end of the
article
article

\begin{abstract}
Combining subspace correction method with least-squares finite element procedure, we construct a new overlapping domain decomposition parallel algorithm for solving the first-order time-dependent convection-diffusion system. This algorithm is fully parallel. We analyze the convergence of approximate solution, and study the dependence of the convergent rate on the spacial mesh size, time increment, iteration number and subdomains overlapping degree. Both theoretical analysis and numerical results suggest that only one or two iterations are needed to reach to given accuracy at each time step.
\end{abstract}

Keywords: Overlapping domain decomposition, Parallel subspace correction, Least-squares, Convection-diffusion system

Mathematics Subject Classification: 65M55, 65M60, 65M12, 65M15

\section{Background}

In this paper, we consider the following initial-boundary value problem for timedependent convection-diffusion system:

$$
\begin{cases}c \frac{\partial u}{\partial t}+\nabla \cdot \sigma+q u=f, & x \in \Omega, 0<t \leq T, \\ \sigma+A \nabla u+\mathbf{b} u=0, & x \in \Omega, 0<t \leq T, \\ u=0, & x \in \Gamma_{D}, \quad 0 \leq t \leq T, \\ \sigma \cdot v=0, & x \in \Gamma_{N}, 0 \leq t,\end{cases}
$$

where $\Omega$ is an open bounded domain $\mathbf{R}^{d}(1 \leq d \leq 3)$, with a Lipschitz continuous boundary $\Gamma=\Gamma_{D} \cup \Gamma_{N}$; and $v$ is the unit vector normal to $\Gamma_{N}$; the flow field $\mathbf{b}=\left(b_{1}, b_{2}, \ldots, b_{d}\right)^{T}$; the source term $q=q(x, t) \geq 0$ and exterior flow function $f=f(x, t)$ are some given functions; the coefficient $c=c(x)$ is positive function and the diffusion coefficient matrix $A=(a(i, j))_{d \times d}$ is a symmetric uniformly positive definite matrix, i.e., there exist some positive constants $c_{*}$ and $a_{*}$ such that

$$
a_{*} \sum_{i=1}^{d} \xi_{i}^{2} \leq \sum_{i, j=1}^{d} a_{i j}(x) \xi_{i} \xi_{j}, \quad c_{*} \leq c(x), \forall \xi \in \mathbf{R}^{d}, x \in \Omega .
$$

This type of partial differential equation arises in many important fields, such as the mathematical modeling of aerodynamics, porous medium fluid flow, fluid dynamics (e.g.

(c) 2016 The Author(s). This article is distributed under the terms of the Creative Commons Attribution 4.0 International License (http://creativecommons.org/licenses/by/4.0/), which permits unrestricted use, distribution, and reproduction in any medium, provided you give appropriate credit to the original author(s) and the source, provide a link to the Creative Commons license, and indicate if changes were made. 
Euler equations, Navier-Stokes equations), meteorology, and semiconductor devices. Many numerical methods have been established to simulate this problem, for example, finite element and finite difference method, Eulerian-Lagrangian localized adjoint method Celia et al. (1990). The streamline diffusion finite element method Hughes and Brooks (1979), least-squares mixed element methods Yang (1999, 2000, 2002), Zhang and Guo (2012), Zhang et al. (2011) and Zhang (2009), and so on. Generally, these numerical procedures result in a large scale of algebraic system, so it is very important and useful to develop effective parallel algorithms both in engineering applications and mathematical analysis.

Recently domain decomposition parallel computation has become a powerful tool for solving a large scale system of partial differential equations. A lot of work has been done on domain decomposition parallel algorithms, for example, see Beilina (2016), Bramble et al. (1990, 1991), Cai (1989), Dolean et al. (2008, 2015), Dryja and Widlund (1987), Lu et al. (1991), Ma et al. (2009), Tarek (2008), Xu (1989, 1992, 2001) and Yang (2010). But many parallel algorithms based on overlapping domain decomposition are iterative algorithms so that many iteration steps are needed to reach given accuracy, which leads to much more global amount of computational work. On the basis of the idea of the parallel subspace correction method proposed by Xu (1989, 1992, 2001), the first author of this paper and Yang established a new parallel algorithm combined with characteristic finite element scheme, finite difference scheme and least-square scheme for one dimensional convection-diffusion problem in Zhang et al. (2011) and Zhang and Yang (2011a, b), where both theoretical analysis and numerical results suggest that when overlapping degree has a positive lower bound independent of mesh size, only one or two iterative times is needed to reach the optimal convergence precision at each time level.

In this paper, using the same technique as in Zhang et al. (2011), Zhang and Yang (2011) and Zhang and Yang (2011), we establish a new parallel algorithm for solving the convection-diffusion system. Here the arbitrary dimensional problem is considered, unlike in Zhang and Yang (2011) only one dimensional model was studied. And the different least-squares finite element scheme from the one in Zhang and Yang (2011) is used to obtain the optimal $L^{2}$-norm error estimate. The partition of unity is applied to distribute the corrections in the overlapping domains reasonably in this parallel algotithm. We analyze the convergence of approximate solution, and study the dependence of the convergent rate on the spacial mesh size, time increment, iteration number and sub-domains overlapping degree. Both theoretical analysis and numerical experiments indicate the full parallelization of the algorithms and very good approximate property.

\section{Parallel algorithm}

Throughout this paper we use usual definitions and notations of Sobolev spaces as in Adams (1975). Let $W^{k, p}(\Omega)(k \geq 0,1 \leq p \leq \infty)$ be Sobolev spaces defined on $\Omega$ with usual norms $\|\cdot\|_{W^{k, p}(\Omega)}$ and $H^{k}(\Omega)=W^{k, 2}(\Omega)$. Define inner products as follows:

$$
\begin{aligned}
(u, v) & =\int_{\Omega} u(x) v(x) d x \quad \forall u, v \in L^{2}(\Omega), \\
(\sigma, \omega) & =\sum_{i=1}^{d}\left(\sigma_{i}, \omega_{i}\right) \quad \forall \sigma, \omega \in\left[L^{2}(\Omega)\right]^{d}, \quad 1 \leq d \leq 3 .
\end{aligned}
$$


Introduce the spaces $\mathcal{W}=\left\{\omega \in\left[L^{2}(\Omega)\right]^{d} ; \nabla \cdot \omega \in L^{2}(\Omega), \omega \cdot v=0\right.$ on $\left.\Gamma_{N}\right\}$ and $\mathcal{V}=$ $\left\{v \in H^{1}(\Omega) ; v=0\right.$ on $\left.\Gamma_{D}\right\}$. Make a time partition $0=t_{0}<t_{1}<\cdots<t_{M-1}<t_{M}=T$ and set $\tau_{n}=t_{n}-t_{n-1}$ and $\tau=\max _{1 \leq n \leq M} \tau_{n}$. Let $w^{n}(x)=w\left(x, t_{n}\right)$. By use of the difference technique with first-order accuracy to discretize the first-order system (1), we can rewrite the system (1) as follows [see Yang (1999)]

$$
\begin{aligned}
c(x) \bar{\partial}_{t} u^{n}(x)+\nabla \cdot \sigma^{n}(x)+q^{n}(x) u^{n}(x) & =f^{n}(x)+R^{n}(x), \quad x \in \Omega, \\
\bar{\partial}_{t} \sigma^{n}(x)+A(x) \nabla \bar{\partial}_{t} u^{n}(x)+\bar{\partial}_{t}\left(\mathbf{b}^{n}(x) u^{n}(x)\right) & =0, \quad x \in \Omega, \\
u^{n}(x) & =0, \quad x \in \Gamma_{D}, \\
\sigma^{n}(x) \cdot v(x) & =0, \quad x \in \Gamma_{N}, \\
u^{0}(x) & =u_{0}(x), \quad x \in \Omega
\end{aligned}
$$

where

$$
\begin{aligned}
R^{n}(x) & =c(x)\left(\bar{\partial}_{t} u^{n}(x)-u_{t}(x)\right)=O\left(\tau_{n} \frac{\partial^{2} u}{\partial t^{2}}\right), \\
\bar{\partial}_{t} u^{n}(x) & =\left(u^{n}-u^{n-1}\right) / \tau_{n} .
\end{aligned}
$$

To construct parallel subspace correction algorithm, we firstly make a domain decomposition. Assume that $\left\{\Omega_{i}^{\prime}\right\}_{i=1}^{N}$ is a non-overlapping domain decomposition of $\Omega$. In order to obtain an overlapping domain decomposition, we extend each subregion $\Omega_{i}^{\prime}$ to a larger region $\Omega_{i}$ such that $\Omega_{i}^{\prime} \subset \Omega_{i} \subset \Omega$ and $\operatorname{dist}\left(\partial \Omega_{i}^{\prime} \backslash \partial \Omega, \partial \Omega_{i} \backslash \partial \Omega\right) \geq H$ for each $1 \leq i \leq N$, where $H>0$ is called as overlapping degree. Let $\mathcal{T}_{h_{u}}$ and $\mathcal{T}_{h_{\sigma}}$ be two families of quasiregular finite element partitions of the domain $\Omega$ such that the elements in the partitions have the diameters bounded by $h_{u}$ and $h_{\sigma}$, respectively. Assume that $\mathcal{T}_{h_{u}, i}=\mathcal{T}_{h_{u}} \cap \Omega_{i}$ and $\mathcal{T}_{h_{\sigma}, i}=\mathcal{T}_{h_{\sigma}} \cap \Omega_{i}$ just are one finite element partition of $\Omega_{i}$ for $1 \leq i \leq N$. Let $\mathcal{W}_{h_{\sigma}} \subset \mathcal{W}$, and $\mathcal{V}_{h_{u}} \subset \mathcal{V}$ be piecewise $r$-degree and $k$-degree polynomial spaces defined on the partitions $\mathcal{T}_{h_{\sigma}}$ and $\mathcal{T}_{h_{u}}$, respectively.

Denote by $\tilde{A}$ the inverse of $A$ and define a bilinear form

$$
\begin{aligned}
a_{n}((\sigma, w),(\omega, v))= & \left(\frac{1}{c}\left(c w+\tau_{n}\left(\nabla \cdot \sigma+q^{n} w\right)\right), c v+\tau_{n}\left(\nabla \cdot \omega+q^{n} v\right)\right) \\
& +\tau_{n}\left(\tilde{A}\left(\sigma+A \nabla w+\mathbf{b}^{n} w\right), \omega+A \nabla v+\mathbf{b}^{n} v\right) .
\end{aligned}
$$

Based on (3) and Yang (1999), we get the standard least-squares finite element procedure: Least-squares scheme Given an initial approximation $\left(\varrho_{h}^{0}, w_{h}^{0}\right) \in \mathcal{W}_{h_{\sigma}} \times \mathcal{V}_{h_{u}}$. For $n=1,2, \ldots, M, \operatorname{seek}\left(\varrho_{h}^{n}, w_{h}^{n}\right) \in \mathcal{W}_{h_{\sigma}} \times \mathcal{V}_{h_{u}}$ such that

$$
\begin{aligned}
a_{n}\left(\left(\varrho_{h}^{n}, w_{h}^{n}\right),\left(\omega_{h}, v_{h}\right)\right) \\
=\left(\frac{1}{c}\left(c w_{h}^{n-1}+\tau_{h} f^{n}\right), c v_{h}+\tau_{h}\left(\nabla \cdot \omega_{h}+q^{n} v_{h}\right)\right) \\
\quad+\tau_{n}\left(\tilde{A}\left(\varrho_{h}^{n-1}+A \nabla w_{h}^{n-1}+\mathbf{b}^{n} w_{h}^{n-1}\right), \omega_{h}+A \nabla v_{h}+\mathbf{b}^{n} v_{h}\right), \\
\quad \forall\left(\omega_{h}, v_{h}\right) \in \mathcal{W}_{h_{\sigma}} \times \mathcal{V}_{h_{u}} .
\end{aligned}
$$

In the following part of this section, we propose the parallel domain decomposition algorithm of the system (4). Define finite element sub-spaces: 


$$
\mathcal{V}_{h_{u}}^{i}=\left\{v_{h} \in \mathcal{V}_{h_{u}} ; \quad v_{h}=0 \text { in } \Omega \backslash \Omega_{\mathrm{i}}\right\}, \quad 1 \leq i \leq N
$$

and

$$
\mathcal{W}_{h_{\sigma}}^{i}=\left\{\sigma_{h} \in \mathcal{W}_{h_{\sigma}} ; \quad \sigma_{h}=0 \text { in } \Omega \backslash \Omega_{\mathrm{i}}\right\}, \quad 1 \leq i \leq N
$$

It is clear that

$$
\mathcal{V}_{h_{u}}=\mathcal{V}_{h_{u}}^{1}+\mathcal{V}_{h_{u}}^{2}+\cdots+\mathcal{V}_{h_{u}}^{N}
$$

and

$$
\mathcal{W}_{h_{\sigma}}=\mathcal{W}_{h_{\sigma}}^{1}+\mathcal{W}_{h_{\sigma}}^{2}+\cdots+\mathcal{W}_{h_{\sigma}}^{N}
$$

It is easily seen that there exists a finite open covering family $\left\{O^{i}\right\}_{i=1}^{N}$ of the domain $\Omega$ such that $O^{i} \cap \Omega \subset \Omega_{i}$. We know that there exists a partition of unity $\left\{\varphi_{i}\right\}_{i=1}^{N}$ (see Toselli and Widlund (2005), Lemma 3.4) such that

(a) $\operatorname{supp}\left(\varphi_{i}\right) \subset O^{i}, \quad 0 \leq \varphi_{i} \leq 1, \quad\left\|\varphi_{i}\right\|_{W^{r, \infty}} \leq C H^{-r}, \quad 1 \leq i \leq N$;

(b) $\varphi_{1}+\varphi_{2}+\cdots+\varphi_{N}=1$ in $\Omega$.

Let $\varphi_{h_{u}}^{i}$ and $\varphi_{h_{\sigma}}^{i}$ be the nodal piecewise linear interpolation of $\varphi_{i}$ on the finite element meshes $\mathcal{T}_{h_{u}}$ and $\mathcal{T}_{h_{\sigma}}$, and $\mathcal{I}_{h_{u}}$ and $\mathcal{I}_{h_{\sigma}}$ be the interpolating operators on $\mathcal{V}_{h_{u}}$ and $\mathcal{W}_{h_{\sigma}}$.

Based on (4), we formulate the parallel subspace correction algorithm.

Parallel algorithm Let $m$ denote the iteration number at each time step. Give an initial approximation $\left(\sigma_{h}^{0}, u_{h}^{0}\right)=\left(\varrho_{h}^{0}, w_{h}^{0}\right) \in \mathcal{W}_{h_{\sigma}} \times \mathcal{V}_{h_{u}}$. For $n=1,2, \ldots, M$, seek $\left(\sigma_{h}^{n}, u_{h}^{n}\right) \in \mathcal{W}_{h_{\sigma}} \times \mathcal{V}_{h_{u}}$ by four steps:

Step 1. Set $\left(\tilde{\sigma}_{0}^{n}, \tilde{u}_{0}^{n}\right)=\left(\sigma_{h}^{n-1}, u_{h}^{n-1}\right)$ and $j:=1$.

Step 2. For $i=1,2, \ldots, N$, seek $\left(\varepsilon_{j}^{i}, e_{j}^{i}\right) \in \mathcal{W}_{h_{\sigma}}^{i} \times \mathcal{V}_{h_{u}}^{i}$, in parallel, such that

$$
\begin{aligned}
a_{n}\left(\left(\varepsilon_{j}^{i}, e_{j}^{i}\right),\left(\omega_{h}, v_{h}\right)\right) \\
=\left(\frac{1}{c}\left(c u_{h}^{n-1}+\tau_{n} f^{n}\right), c \mathcal{I}_{h_{u}}\left(\varphi_{h_{u}}^{i} v_{h}\right)\right. \\
\left.+\tau_{n}\left(\nabla \cdot \mathcal{I}_{h_{\sigma}}\left(\varphi_{h_{\sigma}}^{i} \omega_{h}\right)+q^{n} \mathcal{I}_{h_{u}}\left(\varphi_{h_{u}}^{i} v_{h}\right)\right)\right) \\
+\tau_{n}\left(\tilde{A}\left(\sigma_{h}^{n-1}+A \nabla u_{h}^{n-1}+\mathbf{b}^{n} u_{h}^{n-1}\right), \mathcal{I}_{h_{\sigma}}\left(\varphi_{h_{\sigma}}^{i} \omega_{h}\right)\right. \\
\left.+A \nabla \mathcal{I}_{h_{u}}\left(\varphi_{h_{u}}^{i} v_{h}\right)+\mathbf{b}^{n} \mathcal{I}_{h_{u}}\left(\varphi_{h_{u}}^{i} v_{h}\right)\right) \\
\quad-a_{n}\left(\left(\widetilde{\sigma}_{j-1}^{n}, \widetilde{u}_{j-1}^{n}\right),\left(\mathcal{I}_{h_{\sigma}}\left(\varphi_{h_{\sigma}}^{i} \omega_{h}\right), \mathcal{I}_{h_{u}}\left(\varphi_{h_{u}}^{i} v_{h}\right)\right)\right), \\
\quad \forall\left(\omega_{h}, v_{h}\right) \in \mathcal{W}_{h_{\sigma}} \times \mathcal{V}_{h_{u}} .
\end{aligned}
$$

Step 3. Set corrections

$$
\tilde{\sigma}_{j}^{n}=\tilde{\sigma}_{j-1}^{n}+\sum_{i=1}^{N} \varepsilon_{j}^{i}, \quad \tilde{u}_{j}^{n}=\tilde{u}_{j-1}^{n}+\sum_{i=1}^{N} e_{j}^{i} .
$$


Step 4. If $j<m$, then set $j:=j+1$ and return the step 2; or set

$$
\sigma_{h}^{n}=\tilde{\sigma}_{m}^{n}, \quad u_{h}^{n}=\tilde{u}_{m}^{n}
$$

and then return back to the first step to start iteration at the next time step.

\section{Some lemmas and main result}

In the following sections, we denote by $K$ and $\delta$ some general constants and small positive constants independent of the mesh parameters $H, h_{\sigma} h_{u}$ and $\tau$, which may be different at different occurrences. Let

$$
\begin{aligned}
\|(\omega, v)\|_{a_{n}}^{2}= & \left(\frac{1}{c}\left(c v+\tau_{n}\left(\nabla \cdot \omega+q^{n} v\right)\right), c v+\tau_{n}\left(\nabla \cdot \omega+q^{n} v\right)\right) \\
& +\tau_{n}\left(\tilde{A}\left(\omega+A \nabla v+\mathbf{b}^{n} v\right), \omega+A \nabla v+\mathbf{b}^{n} v\right) .
\end{aligned}
$$

In order to analyze the convergence of parallel algorithm, we introduce projection operators $P_{h_{\sigma}}^{i}: \mathcal{W}_{h_{\sigma}} \rightarrow \mathcal{W}_{h_{\sigma}}^{i}$ and $Q_{h_{u}}^{i}: \mathcal{V}_{h_{u}} \rightarrow \mathcal{V}_{h_{u}}^{i}$ such that

$$
\begin{gathered}
a_{n}\left(\left(P_{h_{\sigma}}^{i} \omega, Q_{h_{u}}^{i} \nu\right),\left(\omega_{h}, v_{h}\right)\right)=a_{n}\left((\omega, v),\left(\omega_{h}, v_{h}\right)\right), \\
\forall\left(\omega_{h}, v_{h}\right) \in \mathcal{W}_{h_{\sigma}}^{i} \times \mathcal{V}_{h_{u}}^{i} i=1,2 \ldots, N .
\end{gathered}
$$

Now, we give some important lemmas which are used to analyze the convergence of parallel algorithm.

We assume that finite element spaces $\mathcal{W}_{h_{\sigma}}$ and $\mathcal{V}_{h_{u}}$ have the inverse property and approximate properties [see Ciarlet (1978)] that there exist some integers $r, r_{1}, k>0$, such that, for $1 \leq q \leq \infty$ and $\forall \omega \in H(\operatorname{div} ; \Omega) \cap\left[W^{r+1, q}(\Omega)\right]^{d}$,

$$
\begin{aligned}
& \inf _{\omega_{h} \in \mathcal{W}_{h_{\sigma}}}\left\|\omega-\omega_{h}\right\|_{\left[L^{q}(\Omega)\right]^{d}} \leq K h_{\sigma}^{r+1}\|\omega\|_{\left[W^{r+1, q}(\Omega)\right]^{d}}, \\
& \inf _{\omega_{h} \in \mathcal{W}_{h_{\sigma}}}\left\|\nabla \cdot\left(\omega-\omega_{h}\right)\right\|_{L^{q}(\Omega)} \leq K h_{\sigma}^{r_{1}}\|\nabla \cdot \omega\|_{W^{r_{1}, q}(\Omega)}, \\
& \inf _{v_{h} \in \mathcal{V}_{h_{u}}}\left\|v-v_{h}\right\|_{L^{q}(\Omega)} \leq K h_{u}^{k+1}\|v\|_{W^{k+1, q}(\Omega)}, \forall v \in L^{2}(\Omega) \cap W^{k+1, q}(\Omega) .
\end{aligned}
$$

Based on Theorem 3.3 in Yang (1999), the following result can be read:

Lemma 1 Let $(\sigma, u)$ and $\left(\varrho_{h}^{n}, w_{h}^{n}\right)$ be the solutions of (1) and least-squares scheme, respectively. Then there holds the a priori error estimate

$$
\max _{n}\left\|u^{n}-w_{h}^{n}\right\|_{L^{2}(\Omega)}+\max _{n}\left\|\sigma^{n}-\varrho_{h}^{n}\right\|_{\left[L^{2}(\Omega)\right]^{d}} \leq K\left\{h_{u}^{k+1}+h_{\sigma}^{r_{1}}+\tau\right\} .
$$

Lemma 2 [See Yang (2001)] For any function $\varphi \in W^{1, \infty}(\Omega)$ and $\omega_{h} \in \mathcal{W}_{h_{\sigma}}$, we have the following estimate

$\left\|\varphi \omega_{h}-\mathcal{I}_{h_{\sigma}}\left(\varphi \omega_{h}\right)\right\|_{\left[L^{2}(\Omega)\right]^{d}} \leq K h_{\sigma} \min \left(\|\varphi\|_{W^{1, \infty}(\Omega)}\left\|\omega_{h}\right\|_{\left[L^{2}(\Omega)\right]^{d}},\|\varphi\|_{H^{1}(\Omega)}\left\|\omega_{h}\right\|_{\left[L^{\infty}(\Omega)\right]^{d}}\right)$

where $d=1,2,3$. 
Lemma 3 For $1 \leq i \leq N$, we have

$$
\begin{gathered}
\left\|\left(\mathcal{I}-\mathcal{I}_{h_{\sigma}}\right)\left(\varphi_{h_{\sigma}}^{i} \omega_{h}\right)\right\|_{\left[L^{2}(\Omega)\right]^{d}} \leq K \frac{h_{\sigma}}{H}\left\|\omega_{h}\right\|_{\left[L^{2}(\Omega)\right]^{d}}, \quad \forall \omega_{h} \in \mathcal{W}_{h_{\sigma}}, \\
\left\|\left(\mathcal{I}-\mathcal{I}_{h_{u}}\right)\left(\varphi_{h_{u}}^{i} v_{h}\right)\right\|_{L^{2}(\Omega)} \leq K \frac{h_{u}}{H}\left\|v_{h}\right\|_{L^{2}(\Omega)}, \quad \forall v_{h} \in \mathcal{V}_{h_{u}} .
\end{gathered}
$$

Proof Using Lemma 2, we know that

$$
\left\|\left(\mathcal{I}-\mathcal{I}_{h_{\sigma}}\right)\left(\varphi_{h_{\sigma}}^{i} \omega_{h}\right)\right\|_{\left[L^{2}(\Omega)\right]^{d}} \leq K h_{\sigma}\left\|\varphi_{h_{\sigma}}^{i}\right\|_{W^{1, \infty}(\Omega)}\left\|\omega_{h}\right\|_{\left[L^{2}(\Omega)\right]^{d}} \leq K \frac{h_{\sigma}}{H}\left\|\omega_{h}\right\|_{\left[L^{2}(\Omega)\right]^{d}} .
$$

This is the first inequality of (8).

In addition, by using the technique of Theorem 3.1 in Yang (2001), we can easily obtain

$$
\begin{aligned}
\|(\mathcal{I}- & \left.\mathcal{I}_{h_{u}}\right)\left(\varphi_{h_{u}}^{i} v_{h}\right) \|_{L^{2}(\Omega)} \\
& \leq K h_{u} \min \left(\left\|\varphi_{h_{u}}^{i}\right\|_{W^{1, \infty}(\Omega)}\left\|v_{h}\right\|_{L^{2}(\Omega)},\left\|\varphi_{h_{u}}^{i}\right\|_{H^{1}(\Omega)}\left\|v_{h}\right\|_{L^{\infty}(\Omega)}\right) \\
& \leq K h_{u}\left\|\varphi_{h_{u}}^{i}\right\|_{W^{1, \infty}(\Omega)}\left\|v_{h}\right\|_{L^{2}(\Omega)} \leq K \frac{h_{u}}{H}\left\|v_{h}\right\|_{L^{2}(\Omega)} .
\end{aligned}
$$

That is the second inequality of (8). The proof of Lemma 3 is completed.

Lemma 4 The following estimate

$$
\begin{aligned}
& \mid a_{n}((\psi, w),(\omega, v))-\sum_{i=1}^{N} a_{n}\left((\psi, w),\left(\mathcal{I}_{h_{\sigma}}\left(\varphi_{h_{\sigma}}^{i} P_{h_{\sigma}}^{i} \omega\right), \mathcal{I}_{h_{u}}\left(\varphi_{h_{u}}^{i} Q_{h_{u}}^{i} v\right)\right) \mid\right. \\
& \quad \leq K\left(\frac{h}{H}+\frac{\sqrt{\tau}}{H}\right)\|(\psi, w)\|_{a_{n}}\left\|\left(\omega_{h}, v_{h}\right)\right\|_{a_{n}}
\end{aligned}
$$

holds for each $(\psi, w)$ and $(\omega, v)$ in $\mathcal{W}_{h_{\sigma}} \times \mathcal{V}_{h_{u}}$.

Proof It is easily seen that

$$
\begin{aligned}
& a_{n}\left((\psi, w),\left(\mathcal{I}_{h_{\sigma}}\left(\varphi_{h_{\sigma}}^{i} P_{h_{\sigma}}^{i} \omega\right), \mathcal{I}_{h_{u}}\left(\varphi_{h_{u}}^{i} Q_{h_{u}}^{i} v\right)\right)\right) \\
& \quad=a_{n}\left((\psi, w),\left(\varphi_{h_{\sigma}}^{i} P_{h_{\sigma}}^{i} \omega, \varphi_{h_{u}}^{i} Q_{h_{u}}^{i} \nu\right)\right) \\
& \quad+a_{n}\left((\psi, w),\left(\left(\mathcal{I}_{h_{\sigma}}-\mathcal{I}\right)\left(\varphi_{h_{\sigma}}^{i} P_{h_{\sigma}}^{i} \omega\right),\left(\mathcal{I}_{h_{u}}-\mathcal{I}\right)\left(\varphi_{h_{u}}^{i} Q_{h_{u}}^{i} \nu\right)\right)\right),
\end{aligned}
$$

and 


$$
\begin{aligned}
a_{n}( & \left.(\psi, w),\left(\varphi_{h_{\sigma}}^{i} P_{h_{\sigma}}^{i} \omega, \varphi_{h_{u}}^{i} Q_{h_{u}}^{i} v\right)\right) \\
= & a_{n}\left(\left(P_{h_{\sigma}}^{i}\left(\varphi_{h_{\sigma}}^{i} \psi\right), Q_{h_{u}}^{i}\left(\varphi_{h_{u}}^{i} w\right)\right),(\omega, v)\right) \\
& +\tau_{n}\left[\left(\frac{1}{c}\left(c w+\tau_{n}\left(\nabla \cdot \psi+q^{n} w\right)\right),\left(P_{h_{\sigma}}^{i} \omega\right) \nabla \varphi_{h_{\sigma}}^{i}\right)\right. \\
& -\left(\frac{1}{c} \psi \nabla \varphi_{h_{\sigma}}^{i}, c Q_{h_{u}}^{i} v+\tau_{n}\left(\nabla \cdot\left(P_{h_{\sigma}}^{i} \omega\right)+q^{n} Q_{h_{u}}^{i} v\right)\right) \\
& +\left(\tilde{A}\left(\psi+A \nabla w+\mathbf{b}^{n} w\right), A \nabla \varphi_{i}^{h} Q_{h_{u}}^{i} v\right) \\
& \left.-\left(\nabla \varphi_{h_{u}}^{i} w, P_{h_{\sigma}}^{i} \omega+A \nabla\left(Q_{h_{u}}^{i} v\right)+\mathbf{b}^{n} Q_{h_{u}}^{i} v\right)\right]
\end{aligned}
$$

and

$$
a_{n}((\psi, w),(\omega, v))=\sum_{i=1}^{N} a_{n}\left(\left(\varphi_{h_{\sigma}}^{i} \psi, \varphi_{h_{u}}^{i} w\right),(\omega, v)\right) .
$$

Hence we have

$$
\begin{aligned}
a_{n}((\psi, w),(\omega, v))-\sum_{i=1}^{N} a_{n}\left((\psi, w),\left(\mathcal{I}_{h_{\sigma}}\left(\varphi_{h_{\sigma}}^{i} P_{h_{\sigma}}^{i} \omega\right), \mathcal{I}_{h_{u}}\left(\varphi_{h_{u}}^{i} Q_{h_{u}}^{i} v\right)\right)\right) \\
=\sum_{i=1}^{N} a_{n}\left(\left(\left(\mathcal{I}-P_{h_{\sigma}}^{i}\right)\left(\varphi_{h_{\sigma}}^{i} \psi\right),\left(\mathcal{I}-Q_{h_{u}}^{i}\right)\left(\varphi_{h_{u}}^{i} w\right)\right),(\omega, v)\right) \\
\quad-\sum_{i=1}^{N} a_{n}\left((\psi, w),\left(\left(\mathcal{I}_{h_{\sigma}}-\mathcal{I}\right)\left(\varphi_{h_{\sigma}}^{i} P_{h_{\sigma}}^{i} \omega\right),\left(\mathcal{I}_{h_{u}}-\mathcal{I}\right)\left(\varphi_{h_{u}}^{i} Q_{h_{u}}^{i} v\right)\right)\right) \\
\quad-\tau_{n} \sum_{i=1}^{N}\left[\left(\frac{1}{c}\left(c w+\tau_{n}\left(\nabla \cdot \psi+q^{n} w\right)\right),\left(P_{h_{\sigma}}^{i} \omega\right) \nabla \varphi_{h_{\sigma}}^{i}\right)\right. \\
\quad-\left(\frac{1}{c} \psi \nabla \varphi_{h_{\sigma}}^{i}, c Q_{h_{u}}^{i} v+\tau_{n}\left(\nabla \cdot\left(P_{h_{\sigma}}^{i} \omega\right)+q^{n} Q_{h_{u}}^{i} v\right)\right) \\
\quad+\left(\tilde{A}\left(\psi+A \nabla w+\mathbf{b}^{n} w\right), A \nabla \varphi_{h_{u}}^{i} Q_{h_{u}}^{i} v\right) \\
\left.-\left(\nabla \varphi_{h_{u}}^{i} w, P_{h_{\sigma}}^{i} \omega+A \nabla\left(Q_{h_{u}}^{i} v\right)+\mathbf{b}^{n} Q_{h_{u}}^{i} v\right)\right] .
\end{aligned}
$$

Noting that

$$
\begin{aligned}
&\left\|\left(\mathcal{I}_{h_{\sigma}}-\mathcal{I}\right)\left(\varphi_{h_{\sigma}}^{i} P_{h_{\sigma}}^{i} \omega\right),\left(\mathcal{I}_{h_{u}}-\mathcal{I}\right)\left(\varphi_{h_{u}}^{i} Q_{h_{u}}^{i} v\right)\right\|_{a_{n}} \\
& \leq K\left\{\left\|c\left(\mathcal{I}_{h_{u}}-\mathcal{I}\right)\left(\varphi_{h_{u}}^{i} Q_{h_{u}}^{i} v\right)\right\|_{L^{2}\left(\Omega_{i}\right)}+\tau_{n}\left\|\nabla \cdot\left(\mathcal{I}_{h_{\sigma}}-\mathcal{I}\right)\left(\varphi_{h_{\sigma}}^{i} P_{h_{\sigma}}^{i} \omega\right)\right\|_{L^{2}\left(\Omega_{i}\right)}\right. \\
&+\tau_{n}\left\|q^{n}\left(\mathcal{I}_{h_{u}}-\mathcal{I}\right)\left(\varphi_{h_{u}}^{i} Q_{h_{u}}^{i} v\right)\right\|_{L^{2}\left(\Omega_{i}\right)}+\sqrt{\tau_{n}}\left[\left\|\left(\mathcal{I}_{h_{\sigma}}-\mathcal{I}\right)\left(\varphi_{h_{\sigma}}^{i} P_{h_{\sigma}}^{i} \omega\right)\right\|_{L^{2}\left(\Omega_{i}\right)}\right. \\
&\left.+\| A \nabla\left(\left(\mathcal{I}_{h_{u}}-\mathcal{I}\right)\left(\varphi_{h_{u}}^{i} Q_{h_{u}}^{i} v\right)\left\|_{L^{2}\left(\Omega_{i}\right)}+\right\| \mathbf{b}^{n}\left(\mathcal{I}_{h_{u}}-\mathcal{I}\right)\left(\varphi_{h_{u}}^{i} Q_{h_{u}}^{i} v\right) \|_{L^{2}\left(\Omega_{i}\right)}\right]\right\} \\
& \leq K\left\{\left(1+\tau_{n}\right) \frac{h_{u}}{H}\left\|Q_{h_{u}}^{i} v\right\|_{L^{2}\left(\Omega_{i}\right)} \tau_{n} \frac{1}{h_{\sigma}} \frac{h_{\sigma}}{H}\left\|P_{h_{\sigma}}^{i} \omega\right\|_{\left[L^{2}\left(\Omega_{i}\right)\right]^{d}}\right. \\
&\left.\quad+\sqrt{\tau_{n}}\left[\frac{h_{\sigma}}{H}\left\|P_{h_{\sigma}}^{i} \omega\right\|_{\left[L^{2}\left(\Omega_{i}\right)\right]^{d}}+\frac{1}{h_{u}} \frac{h_{u}}{H}\left\|Q_{h_{u}}^{i} v\right\|_{L^{2}\left(\Omega_{i}\right)}+\frac{h_{u}}{H}\left\|Q_{h_{u}}^{i} v\right\|_{L^{2}\left(\Omega_{i}\right)}\right]\right\} \\
& \leq K\left(\frac{h}{H}+\frac{\sqrt{\tau}}{H}\right)\left\{\left\|Q_{h_{u}}^{i} v\right\|_{L^{2}\left(\Omega_{i}\right)}+\sqrt{\tau_{n}}\left[\left\|P_{h_{\sigma}}^{i} \omega\right\|_{\left[L^{2}\left(\Omega_{i}\right)\right]^{d}}+\left\|Q_{h_{u}}^{i} v\right\|_{L^{2}\left(\Omega_{i}\right)}\right]\right\} \\
& \leq K\left(\frac{h}{H}+\frac{\sqrt{\tau}}{H}\right)\left\|\left(P_{h_{\sigma}}^{i} \omega, Q_{h_{u}}^{i} v\right)\right\|_{a_{n}, \Omega_{i}},
\end{aligned}
$$


we have

$$
\begin{aligned}
& \left|\sum_{i=1}^{N} a_{n}\left((\psi, w),\left(\left(\mathcal{I}_{h_{\sigma}}-\mathcal{I}\right)\left(\varphi_{h_{\sigma}}^{i} P_{h_{\sigma}}^{i} \omega\right),\left(\mathcal{I}_{h_{u}}-\mathcal{I}\right)\left(\varphi_{h_{u}}^{i} Q_{h_{u}}^{i} v\right)\right)\right)\right| \\
& \quad \leq K\left(\frac{h}{H}+\frac{\sqrt{\tau}}{H}\right)\|(\psi, w)\|_{a_{n}}\left[\sum_{i=1}^{N}\left\|\left(P_{h_{\sigma}}^{i} \omega, Q_{h_{u}}^{i} v\right)\right\|_{a_{n}, \Omega_{i}}^{2}\right]^{1 / 2}, \\
& \quad\left|\sum_{i=1}^{N} a_{n}\left(\left(\left(\mathcal{I}-P_{h_{\sigma}}^{i}\right)\left(\varphi_{h_{\sigma}}^{i} \psi\right),\left(\mathcal{I}-Q_{h_{u}}^{i}\right)\left(\varphi_{h_{u}}^{i} w\right)\right),(\omega, v)\right)\right| \\
& \quad \leq K\left(\frac{h}{H}+\frac{\sqrt{\tau}}{H}\right)\|(\psi, w)\|_{a_{n}}\left[\sum_{i=1}^{N} \|\left(\left(\mathcal{I}-P_{h_{\sigma}}^{i}\right) \omega,\left(\left(\mathcal{I}-\mathcal{I}_{h_{\sigma}}\right)\left(\varphi_{h_{\sigma}}^{i} \psi\right),\left(\mathcal{I}-\mathcal{I}_{h_{u}}^{i}\right)\left(\varphi_{h_{u}}^{i} w\right)\right),\left(\left(\mathcal{I}-P_{h_{\sigma}}^{i}\right) \omega,\left(\mathcal{I}-Q_{a_{n}, \Omega_{i}}^{i}\right]^{1 / 2}\right) v\right)\right) \mid
\end{aligned}
$$

and

$$
\begin{aligned}
& \tau_{n} \sum_{i=1}^{N} {\left[\left|\left(\frac{1}{c}\left(c w+\tau_{n}\left(\nabla \cdot \psi+q^{n} w\right)\right),\left(P_{h_{\sigma}}^{i} \omega\right) \nabla \varphi_{h_{\sigma}}^{i}\right)\right|\right.} \\
&+\left|\left(\frac{1}{c} \psi \nabla \varphi_{h_{\sigma}}^{i}, c Q_{h_{u}}^{i} v+\tau_{n}\left(\nabla \cdot\left(P_{h_{\sigma}}^{i} \omega\right)+q^{n} Q_{h_{u}}^{i} v\right)\right)\right| \\
&+\left(\tilde{A}\left(\psi+A \nabla w+\mathbf{b}^{n} w\right), A \nabla \varphi_{h_{u}}^{i} Q_{h_{u}}^{i} v\right) \mid \\
&\left.+\left|\left(\nabla \varphi_{h_{u}}^{i} w, P_{h_{\sigma}}^{i} \omega+A \nabla\left(Q_{h_{u}}^{i} v\right)+\mathbf{b}^{n} Q_{h_{u}}^{i} v\right)\right|\right] \\
& \leq K \frac{\sqrt{\tau}{ }_{n}}{H}\left[\sum_{i=1}^{N}\|(\psi, w)\|_{a_{n}}\left\|\left(P_{h_{\sigma}}^{i} \omega, Q_{h_{u}}^{i} v\right)\right\|_{a_{n}, \Omega_{i}}^{2}\right]^{1 / 2} .
\end{aligned}
$$

Substituting these estimates into (10) leads to (9). This ends the proof of Lemma 4.

For parallel algorithm, we have the following convergence result:

Theorem 1 Let $(\sigma, u)$ and $\left(\sigma_{h}^{n}, u_{h}^{n}\right)$ are the solutions of the system (1) and parallel algorithm, respectively. If $h^{2 m}=O(\tau)$, then there holds the following a priori error estimate

$$
\begin{aligned}
\max _{n}\left\|u^{n}-u_{h}^{n}\right\|_{L^{2}(\Omega)}+\max _{n}\left\|\sigma^{n}-\sigma_{h}^{n}\right\|_{\left[L^{2}(\Omega)\right]^{d}} \\
\leq K\left\{\left(\frac{h^{2}}{H^{2}}+\frac{\tau}{H^{2}}\right)^{\frac{m}{2}}+h_{u}^{k+1}+h_{\sigma}^{r_{1}}+\tau\right\},
\end{aligned}
$$

where $h=\max \left(h_{\sigma}, h_{u}\right)$.

\section{Proof of Theorem 1}

It is easily seen that parallel algorithm is also equivalent to use an iteration with initial values $\left(\sigma_{h}^{n-1}, u_{h}^{n-1}\right)$ to solve the following equation: $\left(\hat{\sigma}_{h}^{n}, \hat{u}_{h}^{n}\right) \in \mathcal{W}_{h_{\sigma}} \times \mathcal{V}_{h_{u}}$ such that for any $\left(\omega_{h}, v_{h}\right) \in \mathcal{W}_{h_{\sigma}} \times \mathcal{V}_{h_{u}}$ 


$$
\begin{aligned}
a_{n}\left(\left(\hat{\sigma}_{h}^{n}, \hat{u}_{h}^{n}\right),\left(\omega_{h}, v_{h}\right)\right)= & \left(\frac{1}{c}\left(c u_{h}^{n-1}+\tau_{n} f^{n}\right), c v_{h}+\tau_{n}\left(\nabla \cdot \omega_{h}+q^{n} v_{h}\right)\right) \\
& +\tau_{n}\left(\tilde{A}\left(\sigma_{h}^{n-1}+A \nabla u_{h}^{n-1}+\mathbf{b}^{n-1} u_{h}^{n-1}\right), \omega_{h}+A \nabla v_{h}+\mathbf{b}^{n} v_{h}\right) .
\end{aligned}
$$

From (12) we have

$$
\begin{aligned}
a_{n}\left(\left(\sigma_{h}^{n}, u_{h}^{n}\right),\left(\omega_{h}, v_{h}\right)\right)= & \left(\frac{1}{c}\left(c u_{h}^{n-1}+\tau_{n} f^{n}\right), c v_{h}+\tau_{h}\left(\nabla \cdot \omega_{h}+q^{n} v_{h}\right)\right) \\
& +\tau_{n}\left(\tilde{A}\left(\sigma_{h}^{n-1}+A \nabla u_{h}^{n-1}+\mathbf{b}^{n-1} u_{h}^{n-1}\right), \omega_{h}+A \nabla v_{h}+\mathbf{b}^{n} v_{h}\right) \\
& +a_{n}\left(\left(\sigma_{h}^{n}-\hat{\sigma}_{h}^{n}, u_{h}^{n}-\hat{u}_{h}^{n}\right),\left(\omega_{h}, v_{h}\right)\right) .
\end{aligned}
$$

Let $\theta^{n}=u_{h}^{n}-w_{h}^{n}, \rho^{n}=w_{h}^{n}-u^{n}, \pi^{n}=\sigma_{h}^{n}-\varrho_{h}^{n}$ and $\eta^{n}=\varrho_{h}^{n}-\sigma^{n}$. Subtracting (4) from (13), we can get

$$
\begin{aligned}
a_{n}\left(\left(\pi^{n}, \theta^{n}\right),\left(\omega_{h}, v_{h}\right)\right)= & \left(\theta^{n-1}, c v_{h}+\tau_{n}\left(\nabla \cdot \omega_{h}+q^{n} v_{h}\right)\right) \\
& +\tau_{n}\left(\tilde{A}\left(\pi^{n-1}+A \nabla \theta^{n-1}+\mathbf{b}^{n-1} \theta^{n-1}\right), \omega_{h}+A \nabla v_{h}+\mathbf{b}^{n} v_{h}\right) \\
& +a_{n}\left(\left(\sigma_{h}^{n}-\hat{\sigma}_{h}^{n}, u_{h}^{n}-\hat{u}_{h}^{n}\right),\left(\omega_{h}, v_{h}\right)\right) .
\end{aligned}
$$

\section{Lemma 5 For parallel algorithm, we have the estimate}

$$
\left\|\left(\sigma_{h}^{n}-\hat{\sigma}_{h}^{n}, u_{h}^{n}-\hat{u}_{h}^{n}\right)\right\|_{a_{n}} \leq K\left(\frac{h^{2}}{H^{2}}+\frac{\tau}{H^{2}}\right)^{\frac{m}{2}}\left\|\left(\sigma_{h}^{n-1}-\hat{\sigma}_{h}^{n}, u_{h}^{n-1}-\hat{u}_{h}^{n}\right)\right\|_{a_{n}} .
$$

Proof From (5), we have

$$
\begin{aligned}
a_{n}\left(\left(\varepsilon_{j}^{i}, e_{j}^{i}\right),\left(\omega_{h}, v_{h}\right)\right) & =a_{n}\left(\left(\varepsilon_{j}^{i}, e_{j}^{i}\right),\left(P_{h_{\sigma}}^{i} \omega_{h}, Q_{h_{u}}^{i} v_{h}\right)\right) \\
& =a_{n}\left(\left(\hat{\sigma}^{n}-\tilde{\sigma}_{j-1}^{n}, \hat{u}^{n}-\tilde{u}_{j-1}^{n}\right),\left(\mathcal{I}_{h_{\sigma}}\left(\varphi_{h_{\sigma}}^{i} P_{h_{\sigma}}^{i} \omega_{h}\right), \mathcal{I}_{h_{u}}\left(\varphi_{h_{u}}^{i} Q_{h_{u}}^{i} v_{h}\right)\right)\right) .
\end{aligned}
$$

In addition, from parallel algorithm we can obtain the following equation

$$
\begin{aligned}
& a_{n}\left(\left(\tilde{\sigma}_{j}^{n}-\hat{\sigma}_{h}^{n}, \tilde{u}_{j}^{n}-\hat{u}_{h}^{n}\right),\left(\omega_{h}, v_{h}\right)\right) \\
& =a_{n}\left(\left(\tilde{\sigma}_{j-1}^{n}-\hat{\sigma}_{h}^{n}, \tilde{u}_{j-1}^{n}-\hat{u}_{h}^{n}\right),\left(\omega_{h}, v_{h}\right)\right)+a_{n}\left(\left(\sum_{i=1}^{N} \varepsilon_{j}^{i}, \sum_{i=1}^{N} e_{j}^{i}\right),\left(\omega_{h}, v_{h}\right)\right) \\
& =a_{n}\left(\left(\tilde{\sigma}_{j-1}^{n}-\hat{\sigma}_{h}^{n}, \tilde{u}_{j-1}^{n}-\hat{u}_{h}^{n}\right),\left(\omega_{h}, v_{h}\right)\right) \\
& \quad+a_{n}\left(\left(\hat{\sigma}_{h}^{n}-\tilde{\sigma}_{j-1}^{n}, \hat{u}_{h}^{n}-\tilde{u}_{j-1}^{n}\right),\left(\sum_{i=1}^{N} \mathcal{I}_{h_{\sigma}}\left(\varphi_{h_{\sigma}}^{i} P_{h_{\sigma}}^{i} \omega_{h}\right), \sum_{i=1}^{N} \mathcal{I}_{h_{u}}\left(\varphi_{h_{u}}^{i} Q_{h_{u}}^{i} v_{h}\right)\right)\right) .
\end{aligned}
$$

Taking $\left(\omega_{h}, v_{h}\right)=\left(\tilde{\sigma}_{j}^{n}-\hat{\sigma}_{h}^{n}, \tilde{u}_{j}^{n}-\hat{u}_{h}^{n}\right)$ in (17) and using Lemma 4, we have

$$
\left\|\left(\tilde{\sigma}_{j}^{n}-\hat{\sigma}_{h}^{n}, \tilde{u}_{j}^{n}-\hat{u}_{h}^{n}\right)\right\|_{a_{n}}^{2} \leq K\left(\frac{h^{2}}{H^{2}}+\frac{\tau}{H^{2}}\right)\left\|\left(\tilde{\sigma}_{j-1}^{n}-\hat{\sigma}_{h}^{n}, \tilde{u}_{j-1}^{n}-\hat{u}_{h}^{n}\right)\right\|_{a_{n}}^{2} .
$$


Thus, we have

$$
\left\|\left(\tilde{\sigma}_{m}^{n}-\hat{\sigma}_{h}^{n}, \tilde{u}_{m}^{n}-\hat{u}_{h}^{n}\right)\right\|_{a_{n}}^{2} \leq K\left(\frac{h^{2}}{H^{2}}+\frac{\tau}{H^{2}}\right)^{m}\left\|\left(\tilde{\sigma}_{0}^{n}-\hat{\sigma}_{h}^{n}, \tilde{u}_{0}^{n}-\hat{u}_{h}^{n}\right)\right\|_{a_{n}}^{2} .
$$

That is the inequality (15). This ends the proof of Lemma 5.

Hence, we need to estimate the bounds of $\sigma_{h}^{n-1}-\hat{\sigma}_{h}^{n}$ and $u_{h}^{n-1}-\hat{u}_{h^{*}}^{n}$

Lemma 6 For parallel algorithm, we have the following estimate

$$
\begin{aligned}
& \left\|\left(\hat{\sigma}_{h}^{n}-\sigma_{h}^{n-1}, \hat{u}_{h}^{n}-u_{h}^{n-1}\right)\right\|_{a_{n}}^{2} \\
& \quad \leq K \tau_{n}\left\{\int_{t^{n-1}}^{t^{n}}\left\|\left(\frac{\partial \varrho_{h}}{\partial t}, \frac{\partial w_{h}}{\partial t}\right)\right\|_{a_{n}}^{2} \mathrm{~d} t+\tau_{n}\left[\left\|\nabla \cdot \pi^{n-1}\right\|_{L^{2}(\Omega)}^{2}+\left\|\theta^{n-1}\right\|_{L^{2}(\Omega)}^{2}\right]\right\} .
\end{aligned}
$$

Proof From (14) we have

$$
\begin{aligned}
a_{n}( & \left.\left(\hat{\sigma}_{h}^{n}-\sigma_{h}^{n-1}, \hat{u}_{h}^{n}-u_{h}^{n-1}\right),\left(\omega_{h}, v_{h}\right)\right) \\
= & a_{n}\left(\left(\varrho_{h}^{n}-\varrho_{h}^{n-1}, w_{h}^{n}-w_{h}^{n-1}\right),\left(\omega_{h}, v_{h}\right)\right) \\
& -\tau_{n}\left(\frac{1}{c}\left(\nabla \cdot \pi^{n-1}+q^{n} \theta^{n-1}\right), c v_{h}+\tau_{n}\left(\nabla \cdot \omega_{h}+q^{n} v_{h}\right)\right) \\
& \left.-\tau_{n}\left(\tilde{A}\left(\mathbf{b}^{n}-\mathbf{b}^{n-1}\right) \theta^{n-1}\right), \omega_{h}+A \nabla v_{h}+\mathbf{b}^{n} v_{h}\right)
\end{aligned}
$$

Taking $\left(\omega_{h}, v_{h}\right)=\left(\hat{\sigma}_{h}^{n}-\sigma_{h}^{n-1}, \hat{u}_{h}^{n}-u_{h}^{n-1}\right)$ in (21) and using the inequality $a b \leq \frac{1}{\delta} a^{2}+\delta b^{2}$, we can obtain

$$
\begin{aligned}
& \left\|\left(\hat{\sigma}_{h}^{n}-\sigma_{h}^{n-1}, \hat{u}_{h}^{n}-u_{h}^{n-1}\right)\right\|_{a_{n}}^{2} \\
& \leq K \tau_{n}\left\{\int_{t^{n-1}}^{t^{n}}\left\|\left(\frac{\partial \varrho_{h}}{\partial t}, \frac{\partial w_{h}}{\partial t}\right)\right\|_{a_{n}}^{2} \mathrm{~d} t+\tau_{n}\left[\left\|\nabla \cdot \pi^{n-1}\right\|_{L^{2}(\Omega)}^{2}+\left\|\theta^{n-1}\right\|_{L^{2}(\Omega)}^{2}\right]\right\} \\
& \quad+\delta\left\|\left(\hat{\sigma}_{h}^{n}-\sigma_{h}^{n-1}, \hat{u}_{h}^{n}-u_{h}^{n-1}\right)\right\|_{a_{n}}^{2}
\end{aligned}
$$

Hence, when we choose sufficiently small $\delta$, we can obtain the estimate (20). This ends the proof of Lemma 6.

Finally, we prove Theorem 1.

Proof Let $\left(\omega_{h}, v_{h}\right)=\left(\pi^{n}, \theta^{n}-\theta^{n-1}\right)$ in (14), we have

$$
\begin{aligned}
& a_{n}\left(\left(\pi^{n}, \theta^{n}-\theta^{n-1}\right),\left(\pi^{n}, \theta^{n}-\theta^{n-1}\right)\right) \\
&= \tau_{n}\left(\tilde{A}\left(\pi^{n-1}-\pi^{n}\right), \pi^{n}+A \nabla\left(\theta^{n}-\theta^{n-1}\right)+\mathbf{b}^{n}\left(\theta^{n}-\theta^{n-1}\right)\right) \\
&-\tau_{n}\left(\frac{1}{c}\left(\nabla \cdot \pi^{n}+q^{n} \theta^{n-1}\right), c\left(\theta^{n}-\theta^{n-1}\right)+\tau_{n}\left(\nabla \cdot \pi^{n}+q^{n}\left(\theta^{n}-\theta^{n-1}\right)\right)\right) \\
&+\tau_{n}\left(\tilde{A}\left(\mathbf{b}^{n-1}-\mathbf{b}^{n}\right) \theta^{n-1}, \pi^{n}+A \nabla\left(\theta^{n}-\theta^{n-1}\right)+\mathbf{b}^{n}\left(\theta^{n}-\theta^{n-1}\right)\right) \\
&+a_{n}\left(\left(\sigma_{h}^{n}-\hat{\sigma}_{h}^{n}, u_{h}^{n}-\hat{u}_{h}^{n}\right),\left(\pi^{n}, \theta^{n}-\theta^{n-1}\right)\right) .
\end{aligned}
$$


Since

$$
\begin{aligned}
a_{n}\left(\left(\pi^{n}, \theta^{n}-\theta^{n-1}\right),\left(\pi^{n}, \theta^{n}-\theta^{n-1}\right)\right)-\tau_{n}\left(\tilde{A}\left(\pi^{n-1}-\pi^{n}\right), \pi^{n}\right) \\
=\left(c\left(\theta^{n}-\theta^{n-1}\right), \theta^{n}-\theta^{n-1}\right)+\tau_{n}\left[\left(A \nabla\left(\theta^{n}-\theta^{n-1}\right), \nabla\left(\theta^{n}-\theta^{n-1}\right)\right)\right. \\
\quad+\left(\tilde{A} \mathbf{b}^{n}\left(\theta^{n}-\theta^{n-1}\right), \theta^{n}-\theta^{n-1}\right)+\tau_{n}\left(\frac{1}{c} \nabla \cdot \pi^{n}, \nabla \cdot \pi^{n}\right) \\
\left.\quad+\tau_{n}\left(\frac{1}{c} q^{n}\left(\theta^{n}-\theta^{n-1}\right), q^{n}\left(\theta^{n}-\theta^{n-1}\right)\right)\right]+\tau_{n}\left(\tilde{A} \pi^{n}, \pi^{n}\right) \\
\quad+\frac{\tau_{n}}{2}\left[\left(\tilde{A} \pi^{n}, \pi^{n}\right)-\left(\tilde{A} \pi^{n-1}, \pi^{n-1}\right)+\left(\tilde{A}\left(\pi^{n}-\pi^{n-1}\right), \pi^{n}-\pi^{n-1}\right)\right] \\
\quad+2 \tau_{n}\left(\frac{1}{c}\left(c\left(\theta^{n}-\theta^{n-1}\right)+\tau_{n} \nabla \cdot \pi^{n}\right), q^{n}\left(\theta^{n}-\theta^{n-1}\right)\right) \\
\quad+2 \tau_{n}\left(\tilde{A}+\nabla\left(\theta^{n}-\theta^{n-1}\right), \mathbf{b}^{n}\left(\theta^{n}-\theta^{n-1}\right)\right),
\end{aligned}
$$

we have

$$
\begin{aligned}
\left(c\left(\theta^{n}-\theta^{n-1}\right), \theta^{n}-\theta^{n-1}\right)+\tau_{n}\left[\left(A \nabla\left(\theta^{n}-\theta^{n-1}\right), \nabla\left(\theta^{n}-\theta^{n-1}\right)\right)\right. \\
\quad+\left(\tilde{A} \mathbf{b}^{n}\left(\theta^{n}-\theta^{n-1}\right), \theta^{n}-\theta^{n-1}\right)+\tau_{n}\left(\frac{1}{c} \nabla \cdot \pi^{n}, \nabla \cdot \pi^{n}\right) \\
\left.\quad+\tau_{n}\left(\frac{1}{c} q^{n}\left(\theta^{n}-\theta^{n-1}\right), q^{n}\left(\theta^{n}-\theta^{n-1}\right)\right)\right]+\tau_{n}\left(\tilde{A} \pi^{n}, \pi^{n}\right) \\
\quad+\frac{\tau_{n}}{2}\left[\left(\tilde{A} \pi^{n}, \pi^{n}\right)+\left(\tilde{A}\left(\pi^{n}-\pi^{n-1}\right), \pi^{n}-\pi^{n-1}\right)\right] \\
=\frac{\tau_{n}}{2}\left(\tilde{A} \pi^{n-1}, \pi^{n-1}\right)+\tau_{n}\left(\tilde{A}\left(\pi^{n-1}-\pi^{n}\right), A \nabla\left(\theta^{n}-\theta^{n-1}\right)+\mathbf{b}^{n}\left(\theta^{n}-\theta^{n-1}\right)\right) \\
\quad-\tau_{n}\left(\frac{1}{c}\left(\nabla \cdot \pi^{n}+q^{n} \theta^{n-1}\right), c\left(\theta^{n}-\theta^{n-1}\right)+\tau_{n}\left(\nabla \cdot \pi^{n}+q^{n}\left(\theta^{n}-\theta^{n-1}\right)\right)\right) \\
\quad+\tau_{n}\left(\tilde{A}\left(\mathbf{b}^{n-1}-\mathbf{b}^{n}\right) \theta^{n-1}, \pi^{n}+A \nabla\left(\theta^{n}-\theta^{n-1}\right)+\mathbf{b}^{n}\left(\theta^{n}-\theta^{n-1}\right)\right) \\
\quad+a_{n}\left(\left(\sigma_{h}^{n}-\hat{\sigma}_{h}^{n}, u_{h}^{n}-\hat{u}_{h}^{n}\right),\left(\pi^{n}, \theta^{n}-\theta^{n-1}\right)\right) .
\end{aligned}
$$

Next, we estimate the terms on the right-hand side of the error equation (22).

It is clear that

$$
\begin{aligned}
& \tau_{n}\left(\tilde{A}\left(\pi^{n-1}-\pi^{n}\right), A \nabla\left(\theta^{n}-\theta^{n-1}\right)\right) \\
& \quad \leq \frac{\tau_{n}}{2}\left[\left(\tilde{A}\left(\pi^{n}-\pi^{n-1}\right), \pi^{n}-\pi^{n-1}\right)+\left(A \nabla\left(\theta^{n}-\theta^{n-1}\right), \nabla\left(\theta^{n}-\theta^{n-1}\right)\right)\right]
\end{aligned}
$$

and 


$$
\begin{aligned}
& \tau_{n}\left|\left(\tilde{A}\left(\pi^{n-1}-\pi^{n}\right), \mathbf{b}^{n}\left(\theta^{n}-\theta^{n-1}\right)\right)\right|+\tau_{n} \mid\left(\frac{1}{c}\left(\nabla \cdot \pi^{n}+q^{n} \theta^{n-1}\right), c\left(\theta^{n}-\theta^{n-1}\right)\right. \\
&\left.\quad+\tau_{n}\left(\nabla \cdot \pi^{n}+q^{n}\left(\theta^{n}-\theta^{n-1}\right)\right)\right) \mid \\
& \quad+\tau_{n}\left|\left(\tilde{A}\left(\mathbf{b}^{n-1}-\mathbf{b}^{n}\right) \theta^{n-1}, \pi^{n}+A \nabla\left(\theta^{n}-\theta^{n-1}\right)+\mathbf{b}^{n}\left(\theta^{n}-\theta^{n-1}\right)\right)\right| \\
& \quad+\left|a_{n}\left(\left(\sigma_{h}^{n}-\hat{\sigma}_{h}^{n}, u_{h}^{n}-\hat{u}_{h}^{n}\right),\left(\pi^{n}, \theta^{n}-\theta^{n-1}\right)\right)\right| \\
& \leq K \tau_{n}\left\{\left(\frac{h^{2}}{H^{2}}+\frac{\tau}{H^{2}}\right)^{m} \int_{t^{n-1}}^{t^{n}}\left\|\left(\frac{\partial \sigma_{h}}{\partial t}, \frac{\partial w_{h}}{\partial t}\right)\right\|_{a_{n}}^{2} \mathrm{~d} t+\tau_{n}\left[\left\|\theta^{n-1}\right\|_{L^{2}(\Omega)}^{2}\right.\right. \\
&+\left\|\nabla\left(\theta^{n}-\theta^{n-1}\right)\right\|_{\left[L^{2}(\Omega)\right]^{d}}^{2}+\left\|\pi^{n-1}\right\|_{\left[L^{2}(\Omega)\right]^{d}}^{2}+\left\|\pi^{n}\right\|_{\left[L^{2}(\Omega)\right]^{d}}^{2} \\
&\left.\left.+\left\|\nabla \cdot \pi^{n-1}\right\|_{L^{2}(\Omega)}^{2}\right]\right\}+\delta\left[\left\|\theta^{n}-\theta^{n-1}\right\|_{L^{2}(\Omega)}^{2}+\tau_{n}^{2}\left\|\nabla \cdot \pi^{n}\right\|_{L^{2}(\Omega)}^{2}\right] .
\end{aligned}
$$

Substituting the above estimates into (22) and then summing it up from 1 to $n$, we get

$$
\begin{aligned}
& \left\|\pi^{n}\right\|_{\left[L^{2}(\Omega)\right]^{d}}^{2}+\sum_{j=1}^{n} \tau_{j}\left[\left\|\bar{\partial}_{t} \theta^{j}\right\|_{L^{2}(\Omega)}^{2}+\tau_{j}\left\|\nabla \bar{\partial}_{t} \theta^{j}\right\|_{\left[L^{2}(\Omega)\right]^{d}}^{2}+\tau_{j}\left\|\nabla \cdot \pi^{j}\right\|_{L^{2}(\Omega)}^{2}\right] \\
& \leq K\left\{\left(\frac{h^{2}}{H^{2}}+\frac{\tau}{H^{2}}\right)^{m} \int_{0}^{t^{n}}\left\|\left(\frac{\partial \sigma_{h}}{\partial t}, \frac{\partial w_{h}}{\partial t}\right)\right\|_{a_{n}}^{2} \mathrm{~d} t+\sum_{j=1}^{n} \tau_{j}\left[\left\|\pi^{j}\right\|_{\left[L^{2}(\Omega)\right]^{d}}^{2}\right.\right. \\
& \left.\left.\quad+\left\|\theta^{j}\right\|_{L^{2}(\Omega)}^{2}+\tau_{j}^{2}\left\|\nabla \bar{\partial}_{t} \theta^{j}\right\|_{\left[L^{2}(\Omega)\right]^{d}}^{2}\right]\right\} .
\end{aligned}
$$

Applying a known inequality

$$
\left\|\theta^{n}\right\|_{L^{2}(\Omega)}^{2} \leq\left\|\theta^{0}\right\|_{L^{2}(\Omega)}^{2}+\delta \sum_{j=1}^{n} \tau_{j}\left\|\bar{\partial}_{t} \theta^{j}\right\|_{L^{2}(\Omega)}^{2}+K \sum_{j=1}^{n} \tau_{j}\left\|\theta^{j}\right\|_{L^{2}(\Omega)}^{2}
$$

and discrete Gronwall's lemma to (23), we derive that

$$
\max _{n}\left\|\theta^{n}\right\|_{L^{2}(\Omega)}+\max _{n}\left\|\pi^{n}\right\|_{\left[L^{2}(\Omega)\right]^{d}} \leq K\left(\frac{h^{2}}{H^{2}}+\frac{\tau}{H^{2}}\right)^{\frac{m}{2}} .
$$

Using Lemma 1, we can obtain the estimate (11). The proof of Theorem 1 is complete.

\section{Numerical results}

As in Zhang and Yang (2011), we first consider the one dimensional convection-diffusion problem:

$$
\frac{\partial u}{\partial t}-a \frac{\partial^{2} u}{\partial x^{2}}-b \frac{\partial u}{\partial x}+u=f \quad x \in[0,1], \quad 0 \leq t \leq T .
$$

We divide the domain $[0,1]$ into three sub-domains: $\Omega_{1}=\left[0, \frac{1}{3}+\frac{H}{2}\right]$, $\Omega_{2}=\left[\frac{1}{3}-\frac{H}{2}, \frac{2}{3}+\frac{H}{2}\right]$ and $\Omega_{3}=\left[\frac{2}{3}-\frac{H}{2}, 1\right]$, where $H$ is the overlapping degree (see Fig. 1). 


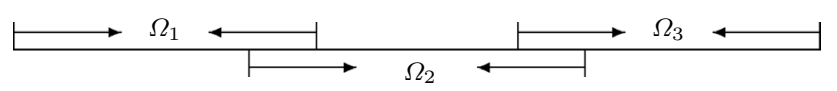

Fig. 1 The sub-domains of $\Omega$

We use piecewise linear polynomial spaces, set $h_{u}=h_{\sigma}=h$ and take the linear unit decomposition functions as in Zhang et al. (2011). We define the $L^{2}$-norm error as follows:

$$
\|(e, E)\|_{2}^{2}=\max _{n}\left\|u^{n}-u_{h}^{n}\right\|_{L^{2}(\Omega)}+\max _{n}\left\|\sigma^{n}-\sigma_{h}^{n}\right\|_{L^{2}(\Omega)},
$$

and the $L^{\infty}$-norm error

$$
\|(e, E)\|_{\infty}=\max _{n}\left(\left|e^{n}\right|,\left|E^{n}\right|\right) .
$$

Experiment I In this experiment, the exact solution is chosen as $u=e^{t} \sin ^{2} \pi x$. Set $T=1$, and $b=1$. For different parameters $a, h, \tau$ and the iterative number $m$ at each time step, we give $L^{2}$-norm errors and the $L^{\infty}$-norm errors in Tables 1, 2 and 3 . These numerical results suggest that we can get a good result for convection-diffusion problem using parallel algorithm, even iterating only one or two cycle at each time step. Moreover, these numerical results also imply that the errors caused by decomposing domain decrease as the discretization parameters $h$ and $\tau$ decrease and increase as the overlapping degree $H$ becomes small, which are coincided with our theoretical result.

\begin{tabular}{|c|c|c|c|c|c|c|c|}
\hline \multirow[t]{2}{*}{$h$} & \multirow[t]{2}{*}{$m$} & \multicolumn{2}{|l|}{$a=1$} & \multicolumn{2}{|l|}{$a=1 e-2$} & \multicolumn{2}{|l|}{$a=1 e-4$} \\
\hline & & $\|\cdot\|_{2}$ & $\|\cdot\|_{\infty}$ & $\|\cdot\|_{2}$ & $\|\cdot\|_{\infty}$ & $\|\cdot\|_{2}$ & $\|\cdot\|_{\infty}$ \\
\hline$\frac{1}{48}$ & $*$ & $2.9866 e-2$ & $1.9250 e-1$ & $8.6118 e-3$ & $2.6493 e-2$ & $7.4344 e-3$ & $1.5421 e-2$ \\
\hline$\frac{1}{48}$ & 1 & $4.0090 e-2$ & $2.0819 e-1$ & $8.5534 e-3$ & $2.5531 e-2$ & $7.4495 e-3$ & $1.4833 e-2$ \\
\hline$\frac{1}{48}$ & 2 & $4.0086 e-2$ & $1.7485 e-1$ & $8.6384 e-3$ & $2.5532 e-2$ & $7.4785 e-3$ & $1.5375 e-2$ \\
\hline$\frac{1}{48}$ & 3 & $4.1043 e-2$ & $1.7967 e-1$ & $8.6403 e-3$ & $2.5532 e-2$ & $7.4790 e-3$ & $1.5379 e-2$ \\
\hline$\frac{1}{48}$ & 4 & $4.1164 e-2$ & $1.7947 e-1$ & $8.6403 e-3$ & $2.5532 e-2$ & $7.4790 e-3$ & $1.5379 e-2$ \\
\hline$\frac{1}{96}$ & $*$ & $1.4800 e-2$ & $9.5488 e-2$ & $4.9541 e-3$ & $2.5220 e-2$ & $3.6348 e-3$ & $7.4154 e-3$ \\
\hline$\frac{1}{96}$ & 1 & $1.7513 e-2$ & $1.0523 e-1$ & $4.9351 e-3$ & $2.4967 e-2$ & $3.6397 e-3$ & $7.3584 e-3$ \\
\hline$\frac{1}{96}$ & 2 & $1.7951 e-2$ & $9.1683 e-2$ & $4.9457 e-3$ & $2.4967 e-2$ & $3.6420 e-3$ & $7.4145 e-3$ \\
\hline$\frac{1}{96}$ & 3 & $1.8186 e-2$ & $9.3327 e-2$ & $4.9458 e-3$ & $2.4967 e-2$ & $3.6420 e-3$ & $7.4147 e-3$ \\
\hline$\frac{1}{96}$ & 4 & $1.8219 e-2$ & $9.3540 e-2$ & $4.9458 e-3$ & $2.4967 e-2$ & $3.6420 e-3$ & $7.4147 e-3$ \\
\hline$\frac{1}{192}$ & $*$ & $7.5407 e-3$ & $4.7396 e-2$ & $2.9959 e-3$ & $2.0611 e-2$ & $1.8231 e-3$ & $4.2963 e-3$ \\
\hline$\frac{1}{192}$ & 1 & $7.9423 e-3$ & $5.1561 e-2$ & $2.9899 e-3$ & $2.0539 e-2$ & $1.8239 e-3$ & $4.2920 e-3$ \\
\hline$\frac{1}{192}$ & 2 & $8.1633 e-3$ & $4.7424 e-2$ & $2.9912 e-3$ & $2.0539 e-2$ & $1.8240 e-3$ & $4.2920 e-3$ \\
\hline$\frac{1}{192}$ & 3 & $8.1986 e-3$ & $4.7096 e-2$ & $2.9912 e-3$ & $2.0539 e-2$ & $1.8240 e-3$ & $4.2920 e-3$ \\
\hline$\frac{1}{192}$ & 4 & $8.2016 e-3$ & $4.7458 e-2$ & $2.9912 e-3$ & $2.0539 e-2$ & $1.8240 e-3$ & $4.2920 e-3$ \\
\hline
\end{tabular}

Table $1 H=\frac{1}{6}, h=\tau$

*The numerical results by least-squares algorithm 
Table $2 H=\frac{1}{12}, h=\tau$

\begin{tabular}{|c|c|c|c|c|c|c|c|}
\hline \multirow[t]{2}{*}{$h$} & \multirow[t]{2}{*}{$m$} & \multicolumn{2}{|l|}{$a=1$} & \multicolumn{2}{|l|}{$a=1 e-2$} & \multicolumn{2}{|l|}{$a=1 e-4$} \\
\hline & & $\|\cdot\|_{2}$ & $\|\cdot\|_{\infty}$ & $\|\cdot\|_{2}$ & $\|\cdot\|_{\infty}$ & $\|\cdot\|_{2}$ & $\|\cdot\|_{\infty}$ \\
\hline$\frac{1}{48}$ & $*$ & $2.9866 e-2$ & $1.9250 e-1$ & $8.6118 e-3$ & $2.6493 e-2$ & $7.4344 e-3$ & $1.5421 e-2$ \\
\hline$\frac{1}{48}$ & 1 & $8.4704 e-2$ & $2.2515 e-1$ & $8.7074 e-3$ & $2.4202 e-2$ & $7.5866 e-3$ & $1.6164 e-2$ \\
\hline$\frac{1}{48}$ & 2 & $8.0447 e-2$ & $1.9139 e-1$ & $8.7352 e-3$ & $2.4202 e-2$ & $7.5212 e-3$ & $1.5371 e-2$ \\
\hline$\frac{1}{48}$ & 3 & $7.9085 e-2$ & $1.8885 e-1$ & $8.7459 e-3$ & $2.4202 e-2$ & $7.5203 e-3$ & $1.5388 e-2$ \\
\hline$\frac{1}{48}$ & 4 & $7.7713 e-2$ & $1.8467 e-1$ & $8.7464 e-3$ & $2.4202 e-2$ & $7.5202 e-3$ & $1.5389 e-2$ \\
\hline$\frac{1}{96}$ & $*$ & $1.4800 e-2$ & $9.5488 e-2$ & $4.9541 e-3$ & $2.5220 e-2$ & $3.6348 e-3$ & $7.4154 e-3$ \\
\hline$\frac{1}{96}$ & 1 & $3.7063 e-2$ & $1.0595 e-1$ & $4.9336 e-3$ & $2.4609 e-2$ & $3.6506 e-3$ & $7.4109 e-3$ \\
\hline$\frac{1}{96}$ & 2 & $3.5576 e-2$ & $8.4116 e-2$ & $4.9480 e-3$ & $2.4609 e-2$ & $3.6484 e-3$ & $7.4152 e-3$ \\
\hline$\frac{1}{96}$ & 3 & $3.5206 e-2$ & $8.2344 e-2$ & $4.9480 e-3$ & $2.4609 e-2$ & $3.6481 e-3$ & $7.4153 e-3$ \\
\hline$\frac{1}{96}$ & 4 & $3.4930 e-2$ & $8.0713 e-2$ & $4.9480 e-3$ & $2.4609 e-2$ & $3.6481 e-3$ & $7.4153 e-3$ \\
\hline$\frac{1}{192}$ & $*$ & $7.5407 e-3$ & $4.7396 e-2$ & $2.9959 e-3$ & $2.0611 e-2$ & $1.8231 e-3$ & $4.2963 e-3$ \\
\hline$\frac{1}{192}$ & 1 & $1.3608 e-2$ & $5.2234 e-2$ & $2.9850 e-3$ & $2.0431 e-2$ & $1.8251 e-3$ & $4.2847 e-3$ \\
\hline$\frac{1}{192}$ & 2 & $1.3257 e-2$ & $4.1956 e-2$ & $2.9875 e-3$ & $2.0431 e-2$ & $1.8249 e-3$ & $4.2847 e-3$ \\
\hline$\frac{1}{192}$ & 3 & $1.3210 e-2$ & $4.2262 e-2$ & $2.9875 e-3$ & $2.0431 e-2$ & $1.8249 e-3$ & $4.2847 e-3$ \\
\hline$\frac{1}{192}$ & 4 & $1.3183 e-2$ & 4.2067e-2 & $2.9875 e-3$ & $2.0431 e-2$ & $1.8249 e-3$ & $4.2847 e-3$ \\
\hline
\end{tabular}

* The numerical results by least-squares algorithm

Table $3 H=\frac{1}{24}, h=\tau$

\begin{tabular}{|c|c|c|c|c|c|c|c|}
\hline \multirow[t]{2}{*}{$h$} & \multirow[t]{2}{*}{$m$} & \multicolumn{2}{|l|}{$a=1$} & \multicolumn{2}{|l|}{$a=1 e-2$} & \multicolumn{2}{|l|}{$a=1 e-4$} \\
\hline & & $\|\cdot\|_{2}$ & $\|\cdot\|_{\infty}$ & $\|\cdot\|_{2}$ & $\|\cdot\|_{\infty}$ & $\|\cdot\|_{2}$ & $\|\cdot\|_{\infty}$ \\
\hline$\frac{1}{48}$ & $*$ & $2.9866 e-2$ & $1.9250 e-1$ & $8.6118 e-3$ & $2.6493 e-2$ & $7.4344 e-3$ & $1.5421 e-2$ \\
\hline$\overline{48}$ & 1 & $2.0573 e-1$ & 4.6896e-1 & $9.1964 e-3$ & $2.8774 e-2$ & $8.0709 e-3$ & $2.1458 e-2$ \\
\hline$\frac{1}{48}$ & 2 & $2.0179 e-1$ & $4.5896 e-1$ & $8.9982 e-3$ & $2.1092 e-2$ & $7.6579 e-3$ & $1.5340 e-2$ \\
\hline$\frac{1}{48}$ & 3 & $2.0061 e-1$ & $4.5878 e-1$ & $9.0836 e-3$ & $2.2172 e-2$ & $7.6797 e-3$ & $1.5379 e-2$ \\
\hline$\frac{1}{48}$ & 4 & $1.9834 e-1$ & $4.5297 e-1$ & $9.1164 e-3$ & $2.2495 e-2$ & $7.6884 e-3$ & $1.5387 e-2$ \\
\hline$\frac{1}{96}$ & $*$ & $1.4800 e-2$ & $9.5488 e-2$ & $4.9541 e-3$ & $2.5220 e-2$ & $3.6348 e-3$ & $7.4154 e-3$ \\
\hline$\frac{1}{96}$ & 1 & $1.1518 e-1$ & $2.8223 e-1$ & $4.8606 e-3$ & $2.3682 e-2$ & $3.6679 e-3$ & $7.6455 e-3$ \\
\hline$\frac{1}{96}$ & 2 & $1.1342 e-1$ & $2.7485 e-1$ & $4.8758 e-3$ & $2.3682 e-2$ & $3.6564 e-3$ & $7.4153 e-3$ \\
\hline$\frac{1}{96}$ & 3 & $1.1266 e-1$ & 2.7333 & $4.8861 e-3$ & $2.3682 e-2$ & $3.6596 e-3$ & $7.4153 e-3$ \\
\hline$\frac{1}{96}$ & 4 & $1.1186 e-1$ & $2.6995 e-1$ & $4.8874 e-3$ & $2.3682 e-2$ & $3.6598 e-3$ & $7.4153 e-3$ \\
\hline$\frac{1}{192}$ & $*$ & $7.5407 e-3$ & $4.7396 e-2$ & $2.9959 e-3$ & $2.0611 e-2$ & $1.8231 e-3$ & $4.2963 e-3$ \\
\hline$\frac{1}{192}$ & 1 & $5.5060 e-2$ & $1.4802 e^{-1}$ & $2.9586 e-3$ & $2.0194 e-2$ & $1.8251 e-3$ & $4.2712 e-3$ \\
\hline$\frac{1}{192}$ & 2 & $5.4280 e-2$ & $1.444 l e-1$ & $2.9635 e-3$ & $2.0194 e-2$ & $1.8259 e-3$ & $4.2712 e-3$ \\
\hline$\frac{1}{192}$ & 3 & $5.3930 e-2$ & $1.4334 e-1$ & $2.9641 e-3$ & $2.0194 e-2$ & $1.8261 e-3$ & $4.2712 e-3$ \\
\hline$\frac{1}{192}$ & 4 & $5.3683 e-2$ & $1.4217 e-1$ & $2.9641 e-3$ & $2.0194 e-2$ & $1.8261 e-3$ & $4.2712 e-3$ \\
\hline
\end{tabular}

* The numerical results by least-squares algorithm 
Experiment II As in Zhang and Yang (2011), we select the right-hand side function with complex structure and the initial condition as follows:

$$
\left\{\begin{array}{l}
f(x, t)=100 e^{t-\frac{x}{5}} \cos (8 \pi x t) \sin ^{2}(7 \pi x) \\
u^{0}(x)=0 .
\end{array}\right.
$$

Choosing $H=1 / 12, h=\tau=1 / 48, b=1$, and $a=1 e-4$, we observe numerical results at different time (see Figs. 2, 3). We use "* " to denote $u_{h}$ and $\sigma_{h}$, the values of the parallel algorithm and use " - " to denote $w_{h}$ and $\varrho_{h}$, the values of least-squares algorithm. These figures clearly show that $u_{h}, \sigma_{h}$ approximate to $w_{h}$ and $\varrho_{h}$ at different time, respectively, which is coincided with our theoretical analysis.

Next, we consider the two dimensional convection-diffusion problem:

$$
\begin{cases}\frac{\partial u}{\partial t}+\nabla \cdot \boldsymbol{\sigma}+u=f, & x \in \Omega, 0<t<T, \\ \boldsymbol{\sigma}+\mathbf{A} \nabla u+\mathbf{b} u=0, & x \in \Omega, 0<t<T,\end{cases}
$$

where $\Omega=[0,1] \times[0,1], \mathbf{A}=a \mathbf{E}, \mathbf{E}$ is the unit matrix, and $\mathbf{b}=(1,1)^{T}$. We divide $\Omega$ into four sub-domains: $\Omega_{1}=[0,0.6] \times[0,0.6], \Omega_{2}=[0.4,1] \times[0,0.6], \Omega_{3}=[0,0.6] \times[0.4,1]$, $\Omega_{4}=[0.4,1] \times[0.4,1]$, see Fig. 4 .

In this section, we use piecewise linear polynomial spaces. And We take the linear unit decomposition functions $\left\{\varphi_{i}\right\}_{i=1}^{4}$ as follows:

$$
\begin{aligned}
& \varphi_{1}(x, y)= \begin{cases}1, & (x, y) \in[0,0.4] \times[0,0.4], \\
3-5 y, & (x, y) \in[0,0.4] \times[0.4,0.6], \\
3-5 x, & (x, y) \in[0.4,0.6] \times[0,0.4], \\
\frac{3}{2}-\frac{5}{4}(x+y), & \text { otherwise }\end{cases} \\
& \varphi_{2}(x, y)= \begin{cases}1, & (x, y) \in[0.6,1] \times[0,0.4], \\
3-5 y, & (x, y) \in[0.6,1] \times[0.4,0.6], \\
5 x-2, & (x, y) \in[0.4,0.6] \times[0,0.4], \\
\frac{1}{4}-\frac{5}{4}(x-y), & \text { otherwise, }\end{cases} \\
& \varphi_{3}(x, y)= \begin{cases}1, & (x, y) \in[0,0.4] \times[0,0.4], \\
5 y-2, & (x, y) \in[0,0.4] \times[0.4,0.6], \\
3-5 x, & (x, y) \in[0.4,0.6] \times[0.6,1], \\
\frac{1}{4}+\frac{5}{4}(y-x), & \text { otherwise }\end{cases} \\
& \varphi_{4}(x, y)= \begin{cases}1, & (x, y) \in[0.6,1] \times[0.6,1], \\
5 y-2, & (x, y) \in[0.6,1] \times[0.4,0.6], \\
5 x-2, & (x, y) \in[0.4,0.6] \times[0.6,1], \\
\frac{5}{4}(x+y)-1, & \text { otherwise. }\end{cases}
\end{aligned}
$$

Experiment III Here we still select the same right-hand side function with complex structure and the initial condition as in Zhang and Yang (2011),

$$
\left\{\begin{array}{l}
f(x, t)=e^{y^{3}-x^{2}-2 t} \sin \left(3 \pi x-6 y+t^{2}\right) \cos (4 \pi y t) \\
u^{0}(x)=0 .
\end{array}\right.
$$



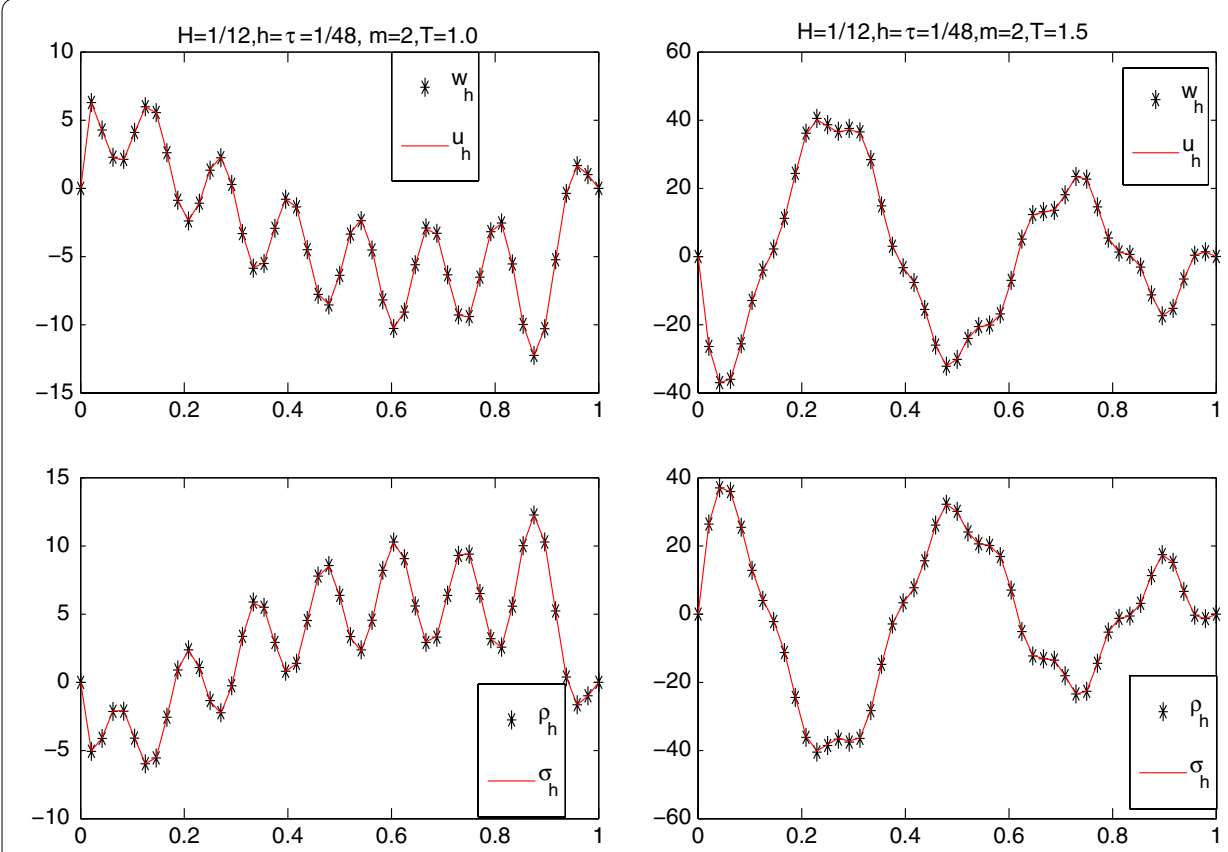

Fig. 2 Numerical results at time $T=1.0,1.5$

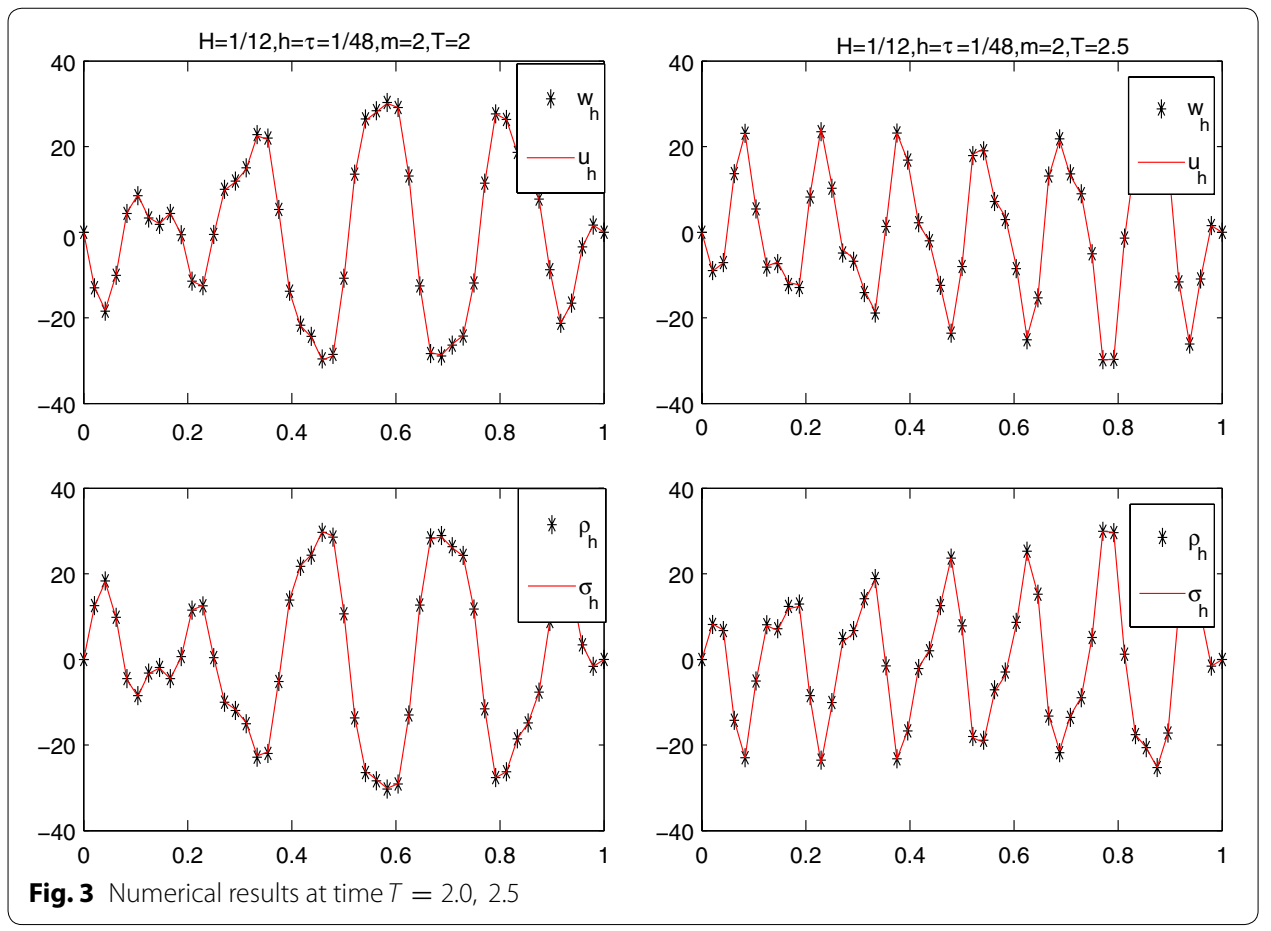

Set $H=0.2, h=\tau=1 / 40$, and $a=1 e-2, T=1.0, m=1$. We can get Figs. 5,6 and 7 . These results suggest that the values $u_{h}, \sigma_{h}=\left(\sigma_{h}^{1}, \sigma_{h}^{2}\right)$ by parallel algorithm approximate to $w_{h}$ and the values $\rho_{h}=\left(\rho_{h}^{1}, \rho_{h}^{2}\right)$ by least-squares scheme respectively, which implies that our method is valid for two-dimensional problem. 

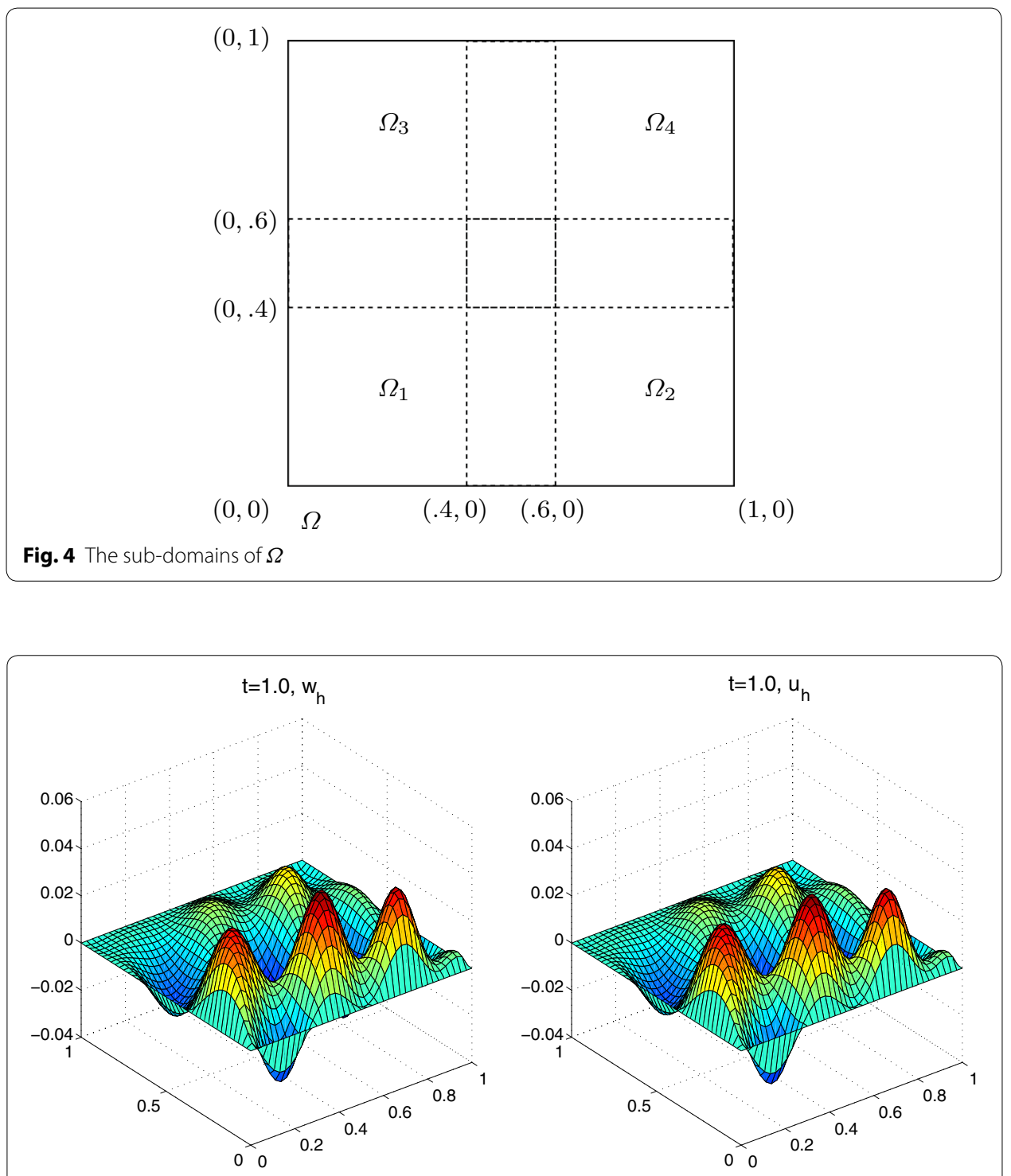

Fig. 5 The values of $w_{h}$ and $u_{h}$ at $T=1.0$

\section{Conclusions}

In this paper, combined subspace correction method with least-squares mixed element procedure, a new class of parallel domain decomposition algorithm is proposed to solve convection-diffusion problem. The convergence of approximate solution, and the dependence of the convergent rate on the spacial mesh size, time increment, iteration number and sub-domains overlapping degree are studied. Both theoretical analysis and numerical experiments indicate the full parallelization of the algorithms and very good approximate property. 

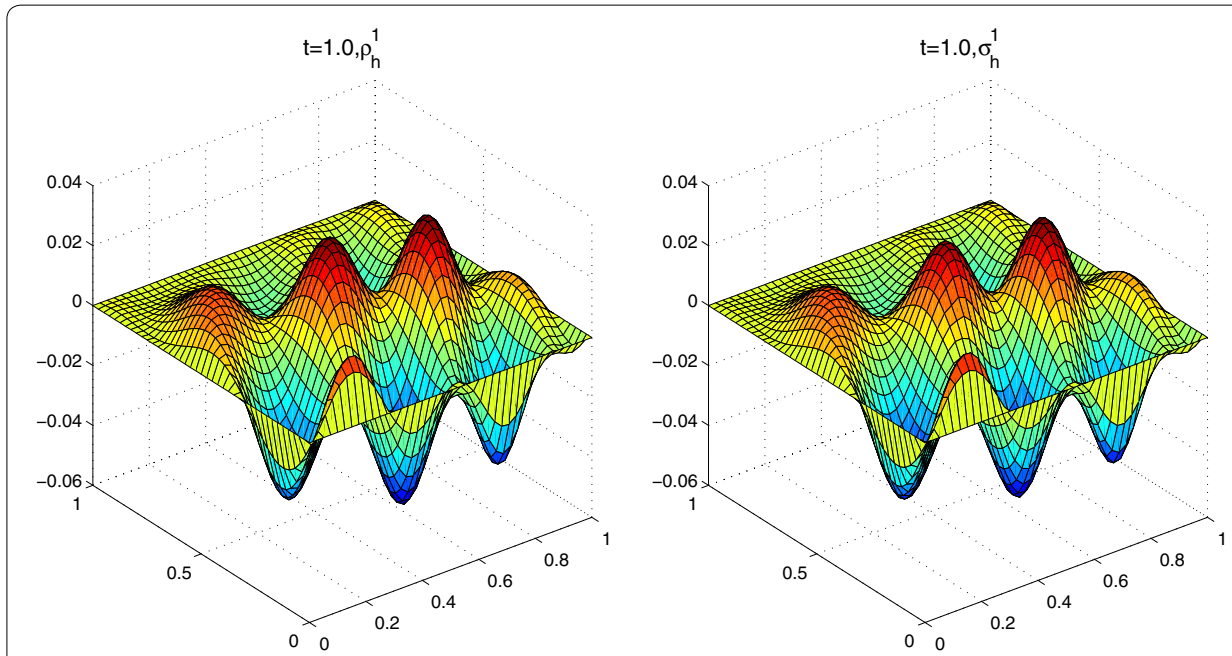

Fig. 6 The values of $\rho_{h}^{1}$ and $\sigma_{h}^{1}$ at $T=1.0$
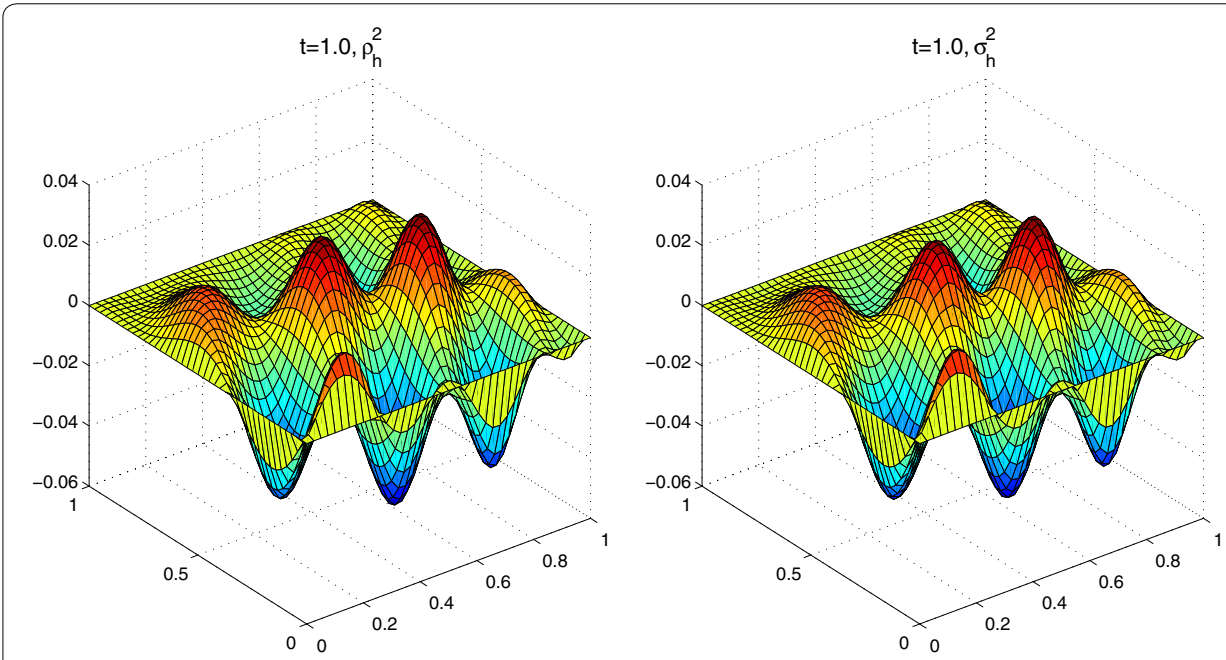

Fig. 7 The values of $\rho_{h}^{2}$ and $\sigma_{h}^{2}$ at $T=1.0$

In fact, though we consider the convection-diffusion problem in this paper, we can extend our method to other complex problems, e.g. saltwater intrusion problem, aerodynamic problems, nuclear waste disposal, etc., which are our future work.

\section{Authors' contributions}

JSZ designed the algorithm and wrote the main manuscript text. HFF and HG did some the experiments and prepared all the figures. All the authors contributed to the discussion of the results. All authors read and approved the final manuscript.

\section{Author details}

'Department of Applied Mathematics, China University of Petroleum, Qingdao 266580, China. ${ }^{2}$ Department of Computational Mathematics, China University of Petroleum, Qingdao 266580, China. ${ }^{3}$ Department of Pure Mathematics, China University of Petroleum, Qingdao 266580, China. ${ }^{4}$ Department of Mathematics, Beijing University of Chemical Technology, Beijing 100029, China.

\section{Acknowledgements}

Jiansong Zhang's work was partially supported by the National Nature Science Foundation of China (11126084, 11401588), China Scholarship Council, and the Natural Science Foundation of Shandong Province (ZR2014AQ005), the Fundamental Research Funds for the Central Universities and the Young Talent Attraction fellowship from the Brazilian 
National Council for Scientific and Technological Development (CNPq). Hui Guo and Hongfei Fu's work was was partially supported by the Fundamental Research Funds for the Central Universities.

\section{Competing interests}

The authors declare that they have no competing interests.

Received: 11 May 2016 Accepted: 20 September 2016

Published online: 01 October 2016

\section{References}

Adams RA (1975) Sobolev spaces. Academic, New York

Beilina L (2016) Domain decomposition finite element/finite difference method for the conductivity reconstruction in a hyperbolic equation. Communications in Nonlinear Science and Numerical Simulation, Elsevier

Bramble JH, Pasciak JE, Xu J (1990) Parallel multilevel preconditioners. Math Comput 55:1-22

Bramble JH, Pasciak JE, Xu J (1991) Convergence estimates for product iterative methods with application to domain decomposition. Math Comput 57:1-21

Cai XC (1989) Some domain decomposition algorithms for nonselfadjont elliptic and parabolic partial differential equations. Ph. D. thesis, Courant Institute

Celia MA, Russell TF, Herrera I, Ewing RE (1990) An Eulerian-Lagrangian localized adjoint method for the advection-diffusion equation. Adv Water Resour 13:187-206

Ciarlet PG (1978) The finite element methods for elliptic problems. North-Holland, New York

Dolean V, Lanteri S, Perrussel R (2008) A domain decomposition method for solving the three-dimensional time-harmonic Maxwell equations discretized by discontinuous Galerkin methods. J Comput Phys 227(3):2044-2072

Dolean V, Jolive P, Nataf F (2015) An introduction to domain decomposition methods: algorithms, theory, and parallel implementation. SIAM

Dryja M, Widlund OB (1987) An additive variant of Schwarz alternating methods for many subregions. Tech. Report 339 Dept. of Comp. Sci. Coutant Institute

Hughes TJR, Brooks AN (1979) A multidimensional upwind scheme with no crosswind diffusion. In: Hughes T.J.R. (Ed.), Finite element methods for convection dominated flows 34:19-35

Lu T, Shih TM, Liem CB (1991) Two synchronous parallel algorithms for partial differential equations. J Comput Math 9(4):74-85

Ma K, Sun T, Yang DP (2009) Parallel Galerkin domain decomposition procedure for parabolic equation on general domain. Numer Methods Partial Differ Equ 25(5):622-636

Tarek M (2008) Domain decomposition methods for the numerical solution of partial differential equations. Lecture Notes in Computational Science and Engineering, Springer

Toselli A, Widlund O (2005) Domain decomposition methods-algorithms and theory. Springer-Verlag, Berlin Heidelberg Xu J (1989) Theory of Multilevel Methods. Ph. D. thesis, Cornell University

Xu J (1992) Iterative methods by space decomposition and subspace correction: A unifying approach. SIAM Review 34:581-613

Xu J (2001) The method of subspace corrections. J Comput Appl Math 128:335-362

Yang DP (1999) Some least-squares Garlerkin procedures for first-order time-dependent convection-diffusion system. Comput Methods Appl Mech Eng 180:81-95

Yang DP (2000) Analysis of least-squares mixed finite element methods for nonlinear nonstationay convection-diffusion problems. Math Comput 69:929-963

Yang DP (2001) A splitting positive definite mixed element method for miscible displacement of compressible flow in porous media. Numer Methods Partial Differ Equ 17:229-249

Yang DP (2002) Least-squares mixed finite element methods for non-linear parabolic problems. J Comput Math 20:153-164

Yang DP (2010) Parallel domain decomposition procedures of improved D-D type for parabolic problems. J Comput Appl Math 233:2779-2794

Zhang JS (2009) Least-squares mixed finite element method for Sobolev equation. Chin J Eng Math 26(4):749-752

Zhang JS, Yang DP, Fu H, Guo H (2011) Parallel characteristic finite element method for time-dependent convection-diffusion problem. Numer Linear Algebra Appl 18(4):695-705

Zhang JS, Yang DP, Zhu J (2011) Two new least-squares mixed finite element procedures for convection-dominated Sobolev equations. Appl Math J Chin Univ 26(4):401-411

Zhang JS, Yang DP (2011) Parallel characteristic finite difference methods for convection-diffusion equations. Numer Methods Partial Differ Equ 27:854-866

Zhang JS, Yang DP (2011) Parallel least-squares finite element method for time-dependent convection-diffusion system. Computing 91:217-240

Zhang JS, Guo H (2012) A split least-squares characteristic mixed element method for nonlinear nonstationary convection-diffusion problem. Int J Comput Math 89(7):932-943 